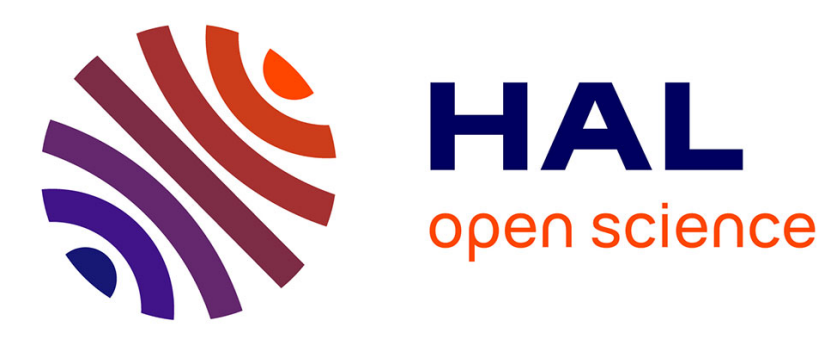

\title{
Financial Markets Equilibrium with Heterogeneous Agents
}

\author{
Jaksa Cvitanic, Elyès Jouini, Semyon Malamud, Clotilde Napp
}

\section{To cite this version:}

Jaksa Cvitanic, Elyès Jouini, Semyon Malamud, Clotilde Napp. Financial Markets Equilibrium with Heterogeneous Agents. Review of Finance, 2012, 16 (1), pp.285-321. 10.1093/rof/rfr018 . halshs00488537

\section{HAL Id: halshs-00488537 https://shs.hal.science/halshs-00488537}

Submitted on 2 Jun 2010

HAL is a multi-disciplinary open access archive for the deposit and dissemination of scientific research documents, whether they are published or not. The documents may come from teaching and research institutions in France or abroad, or from public or private research centers.
L'archive ouverte pluridisciplinaire HAL, est destinée au dépôt et à la diffusion de documents scientifiques de niveau recherche, publiés ou non, émanant des établissements d'enseignement et de recherche français ou étrangers, des laboratoires publics ou privés. 
Swiss Finance Institute

Research Paper Series N ${ }^{\circ} 09$ - 45

\section{Financial M arkets Equilibrium with $\mathrm{H}$ eterogeneous A gents}

Jaksa CVIT ANIC

CALTECH

Elyès JOUINI

U niversité Paris D auphine

Semyon MALAMUD

EPF Lausanne and Swiss Finance Institute

Clotilde NAPP

U niversité Paris D auphine 
Established at the initiative of the Swiss Bankers' Association, the Swiss Finance Institute is a private foundation funded by the Swiss banks and SWX. It merges 3 existing foundations: the International Center FAME, the Swiss Banking School and the Stiftung "Banking and Finance" in Zurich. With its university partners, the Swiss Finance Institute pursues the objective of forming a competence center in banking and finance commensurate to the importance of the Swiss financial center. It will be active in research, doctoral training and executive education while also proposing activities fostering interactions between academia and the industry. The Swiss Finance Institute supports and promotes promising research projects in selected subject areas. It develops its activity in complete symbiosis with the NCCR FinRisk.

The National Centre of Competence in Research "Financial Valuation and Risk Management" (FinRisk) was launched in 2001 by the Swiss National Science Foundation (SNSF). FinRisk constitutes an academic forum that fosters cutting-edge finance research, education of highly qualified finance specialists at the doctoral level and knowledge transfer between finance academics and practitioners. It is managed from the University of Zurich and includes various academic institutions from Geneva, Lausanne, Lugano, St.Gallen and Zurich. For more information see www.nccr-finrisk.ch .

This paper can be downloaded without charge from the Swiss Finance Institute Research Paper Series hosted on the Social Science Research Network electronic library at:

http://ssrn.com/abstract=1520412 


\title{
Financial Markets Equilibrium with Heterogeneous Agents
}

\author{
Jakša Cvitanić, Elyès Jouinił Semyon Malamud ${ }^{\ddagger}$ and Clotilde Napp ${ }^{\S}$
}

December 3, 2009

\begin{abstract}
This paper presents an equilibrium model in a pure exchange economy when investors have three possible sources of heterogeneity. Investors may differ in their beliefs, in their level of risk aversion and in their time preference rate. We study the impact of investors heterogeneity on the properties of the equilibrium. In particular, we analyze the consumption shares, the market price of risk, the risk free rate, the bond prices at different maturities, the stock price and volatility as well as the stock's cumulative returns, and optimal portfolio strategies. We relate the heterogeneous economy with the family of associated homogeneous economies with only one class of investors. We consider cross sectional as well as asymptotic properties.

Keywords: equilibrium, heterogeneous agents, volatility, optimal portfolios, survival, yield curve, long yield
\end{abstract}

JEL Classification. D53, G11, G12

\section{Introduction}

This paper presents an equilibrium model in a pure exchange economy when investors have three possible sources of heterogeneity. Investors may differ in

${ }^{*}$ Caltech, Division of Humanities and Social Sciences, M/C 228-77, 1200 E. California Blvd. Pasadena, CA 91125. Ph: (626) 395-1784. E-mail: cvitanic@hss.caltech.edu

†Université Paris Dauphine. E-mail:jouini@ceremade.dauphine.fr

${ }^{\ddagger}$ EPF Lausanne and Swiss Finance Institute. E-mail: semyon.malamud@epfl.ch

$\S$ Université Paris Dauphine. E-mail:Clotilde.Napp@dauphine.fr 
their beliefs, in their level of risk aversion and in their time preference rate. We study the impact of investors heterogeneity on the equilibrium properties.

The present paper is the first to deal with the three aspects of heterogeneity simultaneously, which enables us to better identify the impact of heterogeneity and its determinants. We analyze agents interactions, and the impact of heterogeneity at the individual level, in particular on individual consumption, individual valuations, individual portfolio holdings and risk sharing rules. At the aggregate level, we analyze properties of the market price of risk, of the risk free rate, of the bond prices, and of the stock price and volatility. We compare equilibrium characteristics to the characteristics in the homogeneous economies populated by one class of agents only. We consider cross sectional as well as asymptotic properties.

Heterogeneity implies that investors value differently the states of the world. Individual valuations of the states of the world are reflected by individual Arrow-Debreu prices, i.e., the Arrow-Debreu prices that would prevail in the economy populated by one type of agents only. For example, a pessimistic agent values more than an optimistic agent the states of the world where the level of aggregate consumption is low (or the Arrow-Debreu assets associated to those bad states), as she assigns a higher probability to those states. Analogously, a more risk averse agent values more than a less risk averse agent the states of the world with a low level of aggregate consumption. Finally, a more patient agent values more the states of the world in the distant future. More generally, the exact mixture of those heterogeneous individual parameters of beliefs, risk aversion and time preference determines individual valuations.

We analyze how heterogeneous individual valuations impact the equilibrium valuation, i.e., the Arrow-Debreu prices. We first obtain that if individual Arrow-Debreu prices are decreasing in aggregate endowment, then the equilibrium Arrow-Debreu price at the aggregate level is also decreasing in aggregate endowment. At all dates and in all states of the world, the ArrowDebreu price lies in the range bounded by the minimum individual ArrowDebreu price and the maximum individual Arrow-Debreu price. When a given individual Arrow-Debreu price dominates the others, then it governs the equilibrium Arrow-Debreu price at the aggregate level. This means that when a class of agents values much more than the others an Arrow-Debreu asset, that class drives the market for that asset. We find that in "extreme" states of the world (very high or very low level of aggregate endowment, in the distant future), one agent dominates individual valuations of Arrow- 
Debreu assets. This "dominating" agent is not the same asymptotically, in very good or in very bad states of the world. Distinct classes of agents drive the price of the different Arrow-Debreu assets.

We find that for very high (low) level of aggregate endowment, the equilibrium Arrow-Debreu price is determined by the agent with the highest (lowest) individual market price of risk where the individual market price of risk is defined as the market price of risk that would prevail in an economy populated by one class of agents only ${ }^{1}$. For example, if there is heterogeneity in beliefs (risk aversion levels) only, the equilibrium Arrow-Debreu price for very low levels of aggregate consumption is given by the Arrow-Debreu price of the most pessimistic (most risk averse) agent. That agent is the one who values the most the Arrow-Debreu asset associated to these "bad" states. We show that in the distant future the agent with the lowest survival index ${ }^{2}$ (Yan, 2008) governs the Arrow-Debreu price. In the case with heterogeneity in beliefs (time preference) only, this agent is the most rational (patient) agent.

As for consumption, an agent consumes relatively more in the states of the world that she finds relatively more desirable, as her individual Arrow-Debreu price is higher, and so is her individual consumption. As a consequence, the results about the Arrow-Debreu prices can be applied to the consumption shares. In particular, only the class of agents with the highest market price of risk (lowest market price of risk, lowest survival index) dominates the market in the sense of the consumption share in very bad states of the world (in very good states of the world, asymptotically). We also retrieve the result of Yan (2008) that only the agent with the lowest survival index survives in the long run.

Fluctuations across time and states of the world in the individual consumptions and hence in the individual risk tolerance levels ${ }^{3}$ generate fluc-

\footnotetext{
${ }^{1}$ The individual market price of risk of agent $i$ is given by $\theta_{i}=\gamma_{i} \sigma-\delta_{i}$ where $\gamma_{i}, \delta_{i}$ and $\sigma$ respectively denote the individual level of risk aversion, the individual level of optimism and the volatility of aggregate endowment. The individual (required) market price of risk reflects the agent's motives to invest in a risky asset. It increases with the level of risk aversion and with the level of pessimism.

${ }^{2}$ The survival index of agent $i$ is defined by $\kappa_{i} \equiv \rho_{i}+\gamma_{i}\left(\mu-\frac{\sigma^{2}}{2}\right)+\frac{1}{2} \delta_{i}^{2}$, where $\rho_{i}, \gamma_{i}, \delta_{i}, \mu$ and $\sigma$ respectively denote the individual level of time preference, risk aversion, optimism and the drift and volatility of aggregate endowment.

${ }^{3}$ Letting $c_{i t}$ denote the individual equilibrium consumption level of agent $i$, the individual consumption share of agent $i$ is defined by $\frac{c_{i t}}{\sum_{j=1}^{N} c_{j t}}$. The relative level of absolute risk
} 
tuations in the behavior at the aggregate level (beliefs, risk aversion and patience). Indeed, the aggregate parameters can be written as a risk tolerance weighted average of the individual parameters. We obtain time and state varying levels of risk aversion, optimism and patience at the aggregate level even though the individual levels of risk aversion, optimism and patience are constant. This generates at the aggregate level waves of pessimism/optimism, of risk aversion and of patience. For example, in bad states of the world, more pessimistic agents have a higher consumption share and a higher relative level of risk tolerance, which leads to a pessimistic bias at the aggregate level. Without referring to irrational behavior, this can explain excess of pessimism in periods of recession. Analogously, we get that more risk averse agents have a higher weight in the economy in periods of recession, which leads to more risk aversion at the aggregate level. This is interesting in relation to Brunnermeier and Nagel (2008), who show that individual risk aversion is not time varying even though fluctuating (and in particular countercyclical) risk aversion, as in habit preference models (Campbell and Cochrane, 1999), would help matching aggregate data.

The market price of risk is the risk tolerance weighted average of the individual market prices of risk. While the individual market prices of risk are constant in time and state of the world, the equilibrium market price of risk in the heterogeneous economy fluctuates in time and state of the world between the two bounds which are the lowest individual market price of risk and the highest individual market price of risk. If one class of agents dominates the level of risk tolerance, then the market price of risk in the heterogeneous economy is dominated by their market price of risk. Applying the results on the risk tolerance, we get that for very high (low) levels of aggregate consumption, the market price of risk is given by the lowest (highest) market price of risk, and that the market price of risk converges asymptotically to the market price of risk of the surviving agent. For example, when there is heterogeneity in beliefs only, the most pessimistic agents dominate the economy for low levels of aggregate endowment leading to the highest market price of risk.

Moreover, we obtain that the market price of risk is countercyclical: the higher the aggregate endowment the lower the market price of risk. This result is quite striking, since it holds for any distribution of the parame-

tolerance of agent $i$ is given by $\omega_{i t} \equiv-\frac{u_{i}^{\prime}}{u_{i}^{\prime \prime}}\left(c_{i t}\right)\left[\sum-\frac{u_{i}^{\prime}}{u_{i}^{\prime \prime}}\left(c_{i t}\right)\right]^{-1}=\frac{\left(1 / \gamma_{i}\right) c_{i t}}{\sum_{j=1}^{N}\left(1 / \gamma_{j}\right) c_{j t}}$, where $\gamma_{i}$ denotes the risk aversion level of agent $i$. 
ters of risk aversion and beliefs. It is heterogeneity and its impact on the fluctuations of the relative levels of risk tolerance which generates this countercyclical behavior; indeed, an increase in aggregate endowment leads to higher relative levels of risk tolerance for the agents with a high market price of risk. This countercyclicality property is consistent with observations that "equity risk premia seem to be higher at business troughs than they are at peaks" (Campbell and Cochrane, 1999).

Contrary to the market price of risk, the risk free rate is not a weighted average of the individual risk free rates $^{4}$. In general the equilibrium characteristics in the heterogeneous economy (with $N$ classes of agents) are fundamentally different from the equilibrium characteristics in a homogeneous economy. The equilibrium risk free rate can lie outside the range bounded by the lowest individual risk free rate and by the highest individual risk free rate. However, in "extreme" states of the world, the risk free rate behaves as a risk tolerance weighted average of the individual ones: the risk free rate for very high (low) levels of aggregate endowment is given by the individual risk free rate of the agent with the lowest (highest) market price of risk and the asymptotic risk free rate is given by the risk free rate of the surviving agent.

The equilibrium long term bond yield is given by the individual long term bond yield of the agent with the highest savings motives (lowest individual risk free rate). The reasoning is the same as for the Arrow-Debreu assets: when a given class of agents has an individual price for an asset that dominates the other individual prices, then that class of agents governs the equilibrium price in the heterogeneous economy. The agent with the highest savings motives values the most (very) long term bonds. Hence, the individual zero-coupon bond price of that agent asymptotically dominates the other individual bond prices. The agent with the highest savings motives hence governs the long term bond yield in the heterogeneous economy.

Note that the agent with the highest savings motives differs from the agent with the lowest survival index. It is quite striking that the agent who drives the asymptotic long term bond yield differs from the agent who drives the asymptotic risk free rate even though the bond yield is an average of the

\footnotetext{
${ }^{4}$ The individual risk free rate is the risk free rate that would prevail if the economy was populated by one agent only. The individual risk free rate of agent $i$ is given by $r_{i}=\rho_{i}+\gamma_{i}\left(\mu+\delta_{i}\right)-\frac{1}{2} \gamma_{i}\left(\gamma_{i}+1\right) \sigma^{2}$, where $\rho_{i}, \gamma_{i}, \delta_{i}, \mu$ and $\sigma$ respectively denote the individual level of time preference, risk aversion, the individual level of optimism, the drift and the volatility of aggregate endowment.
} 
risk free rates. In particular, in the long run, the yield curve is driven by the risk free rate of the agent with the lowest survival index at one end of the yield curve, whereas at the other end, it is driven by the risk free rate of the agent with the highest savings motives or equivalently the lowest risk free rate. In fact we show that for times $t$ far in the future, there are distinct time periods during which distinct agents drive the long term bond yield as seen from time $t$. The (asymptotic) yield curve is defined stepwise, and each subinterval is associated with a given agent in the sense that the marginal rate on that subinterval corresponds to the rate in the economy made of this agent only. The same reasoning as for the Arrow-Debreu assets and the long term bond still holds since the agent who makes the market of the zero coupon bond on the considered subinterval is the agent who values the most this zero coupon bond. She is characterized by a maximization program involving a weighted average of the savings motives index and of the survival index. In the short run, the whole weight is on the savings motives index and in the long run, the whole weight is on the survival index. In between, the agent who governs the asymptotic yield curve maximizes a trade off between the savings motives and survival. For example, when there is only heterogeneity in beliefs, one end of the asymptotic yield curve is dominated by the most rational agent (maximization of the survival motives), the other end is dominated by the most pessimistic agent (maximization of the savings motives) and in the middle, the asymptotic yield curve is governed, in intervals, by more and more pessimistic agents (maximization of a trade off between rationality and pessimism). Note that in this setting, only pessimistic agents impact the asymptotic yield curve.

These results are in line with Preferred Habitat Theory (Modigliani and Sutch, 1966), in which each agent has a preferred habitat corresponding to her natural investment horizon. In our framework, this investment horizon naturally emerges for each agent, as the result of heterogeneity.

This implies in particular that survival and long run impact are different concepts. We are in a setting where only the agent with the lowest survival index survives (and has an impact on prices in the sense of Kogan et al., 2008), however non surviving agents do have an impact on asset prices, even in the long run. In the case of the yield curve, one may argue that this impact is at more and more distant horizons, but we also provide examples where the impact of non surviving agents does not vanish asymptotically. As far as risky assets are concerned, we also show that the long run return of these assets are impacted by nonsurviving agents and we provide an example 
where the agent who drives the long run discount rate is different from the agent who drives the long run risky returns and both of them are different from the surviving agent who drives the instantaneous risk free rate in the long run.

We also analyze the behavior of stock volatility, which converges to dividend volatility. Asymptotically, only the surviving agent is present (in terms of consumption shares or risk tolerance levels) and the stock volatility in the heterogeneous economy converges to the surviving agent's individual stock volatility, which is the dividend volatility. We show that for finite times stock volatility fluctuates between bounds determined by the maximal difference between market prices of risk associated with different agents.

We get similar bounds for the optimal portfolios. If all risk aversions are larger than one, then in the limit the optimal portfolios are driven by the market price of risk associated with the surviving agent.

We now discuss additional related works. Dumas (1989) and Wang (1996) are among the first papers to study equilibrium in the markets with two heterogeneous agents (heterogeneous in risk aversion levels). Bhamra and Uppal (2009a) consider the same economy, and derive conditions under which excess volatility is positive. ${ }^{5}$ Bhamra and Uppal (2009b) derive closed form expressions as convergent power series for an economy populated by two CRRA agents with arbitrary risk aversions, discount rates and heterogeneous beliefs. Dumas, Kurshev and Uppal (2009) also study excess volatility and non-myopic optimal portfolios in a model with two CRRA agents with identical preferences but heterogeneous beliefs. An economy with heterogeneous beliefs with two logarithmic agents who adapt their beliefs dynamically and trade subject to portfolio constraints is studied in Detemple and Murthy (1997). Jouini and $\operatorname{Napp}$ (2007, 2009) and Jouini et al. (2008) consider heterogeneous beliefs, but homogenous risk aversion. Kogan et al. (2006) also consider a two agent economy with agents maximizing utility only from terminal consumption and get asymptotic results when horizon increases on the impact of non surviving agents on asset prices. They pose as an open question what the analogue of their result is when the agents maximize intermediate consumption. We provide answers to that question. From a quite different perspective and in a more general setting, Kogan et al (2008) pro-

\footnotetext{
${ }^{5}$ The main message of Bhamra and Uppal (2009a) is that allowing the agents to trade in an additional derivative security, making the market complete, may actually increase the market volatility. Because of completeness, their equilibrium coincides with the ArrowDebreu equilibrium of Wang (1996).
} 
vide a typology of the different possible asymptotic behaviors in terms of survival and price impact. Berrada (2008) studies the consumption shares of several CRRA agents with same risk aversion and different beliefs, and most results are obtained numerically. Cvitanić and Malamud (2009a,b,c) consider equilibrium with many traders maximizing utility only from terminal consumption. Berrada et al. (2007) provide necessary and sufficient conditions for zero equilibrium trading volume in a general continuous-time model with heterogeneous agents, multiple goods, and multiple securities. Yan (2008) first introduced the same notion of the survival index that we use, and obtains the result on the domination of the surviving agent in terms of consumption shares. Sandroni (2000) and Blume and Easley (2006) study survival with another definition of the survival index and in a model different from ours.

\section{The Model}

We consider a continuous-time Arrow-Debreu economy with an infinite horizon, in which heterogeneous agents maximize their expected utility from future consumption.

Uncertainty is described by a one-dimensional, standard Brownian motion $W_{t}, t \in[0, \infty)$ on a complete probability space $(\Omega, \mathcal{F}, P)$, where $\mathcal{F}$ is the augmented filtration generated by $W_{t}$. There is a single consumption good and we denote by $D$ the aggregate dividend or endowment process. We make the assumption that $D$ satisfies the following stochastic differential equation

$$
d D_{t}=\mu D_{t} d t+\sigma D_{t} d W_{t} \quad D_{0}=1
$$

where the mean growth rate $\mu$ and the volatility $\sigma$ are constants.

There are $N$ (types of) agents indexed by $i=1, \ldots, N$. Agents have different expectations about the future of the economy. More precisely, agents disagree about the mean growth rate. We denote by $\mu_{i}$ the mean growth rate anticipated by agent $i$. Letting

$$
\delta_{i} \equiv \frac{\mu_{i}-\mu}{\sigma}
$$

denote agent $i$ 's error in her perception of the growth of the economy normalized by its risk $^{6}$, we introduce the probability measure $P^{i}$ defined by

\footnotetext{
${ }^{6}$ The parameter $\delta_{i}$ also represents the difference between agent $i$ 's perceived Sharpe ratio and the true one.
} 
its density with respect to $P$ given by $Z_{i t}=e^{\delta_{i} W_{t}-\frac{1}{2} \delta_{i}^{2} t}$. From agent $i$ point of view, the aggregate endowment process satisfies the following stochastic differential equation

$$
d D_{t}=\mu_{i} D_{t} d t+\sigma D_{t} d W_{t}^{i} \quad D_{0}=1
$$

where, by Girsanov Theorem, $W_{t}^{i} \equiv W_{t}-\delta_{i} t$ is a Brownian motion with respect to $P^{i}$. Note that agents are persistent in their mistakes: the probability measures $P^{i}$ may represent erroneous beliefs as well as behavioral biases like optimism or pessimism.

Agent $i$ 's utility function is given by $u_{i}(c)=\frac{c^{1-\gamma_{i}-1}}{1-\gamma_{i}}$ for $\gamma_{i}>0$, where $\gamma_{i}$ is the relative risk aversion coefficient. In the following, we let $b_{i} \equiv \frac{1}{\gamma_{i}}$ denote the relative risk tolerance of agent $i$. Agent $i$ 's time preference rate is denoted by $\rho_{i}$.

Agent $i$ 's utility for a given consumption stream $\left(c_{t}\right)$ is then given by

$$
E^{P^{i}}\left[\int_{0}^{\infty} e^{-\rho_{i} t} u_{i}\left(c_{t}\right) d t\right]
$$

where $E^{P^{i}}$ denotes the expectation operator from agent $i$ 's perspective. There are then in our setting three possible sources of heterogeneity among agents: heterogeneity in beliefs, heterogeneity in time preference rates and heterogeneity in risk aversion levels.

Agents have endowments denoted by $\left(e^{*^{i}}\right)$ with $\sum_{i=1}^{N} e^{*^{i}}=D$. We assume that markets are complete which means that all Arrow-Debreu securities can be traded. A state price density (or stochastic discount factor) $M$ is a positive process such that $M(t, \omega)$ corresponds to the price of the asset that pays one dollar at date $t$ and in state $\omega$. For a given state price density $M$, agent $i$ 's intertemporal optimization program is given by

$$
\left(O_{i M}\right): \max _{c}\left\{E^{P^{i}}\left[\int_{0}^{\infty} e^{-\rho_{i} t} u_{i}\left(c_{t}\right) d t\right] \mid E\left[\int_{0}^{\infty} M_{t}\left(c_{t}-e_{t}^{*^{i}}\right) d t\right] \leq 0\right\} .
$$

We adopt the usual definition of equilibrium.

Definition 2.1 An equilibrium is a state price density $M$ and consumption processes $\left(c_{i t}\right)$ such that each consumption process $\left(c_{i t}\right)$ solves agent $i$ 's optimization program $\left(O_{i M}\right)$ and markets clear, i.e. $\sum_{i=1}^{N} c_{i t}=D_{t}$. 
We assume that such an equilibrium exists and in the next we let $M$ (resp. $c_{i t}$ ) denote the equilibrium state price density (resp. the equilibrium consumption processes).

In order to deal with asset pricing issues, we suppose that agents can continuously trade in a riskless asset and in risky stocks ${ }^{7}$. We let $S^{0}$ denote the riskless asset price process with dynamics $d S_{t}^{0}=r_{t} S_{t}^{0} d t$, the parameter $r$ denoting the risk free rate. Since there is only one source of risk, all risky assets have the same instantaneous Sharpe ratio and it suffices to focus on one specific risky asset. We consider the asset $S$ whose dividend process is given by the total endowment of the economy and we denote respectively by $\mu_{S}$ and $\sigma_{S}$ its drift and volatility. We let

$$
\theta \equiv \frac{\mu_{S}+D_{t} S_{t}^{-1}-r}{\sigma_{S}}
$$

denote the asset's Sharpe ratio or equivalently the market price of risk. The parameters $r, \mu_{S}$ and $\sigma_{S}$ are to be determined endogenously in equilibrium.

We let $B(t, T)$ denote the price at time $t$ of the pure-discount bond price delivering 1 dollar at time $T$, i.e.,

$$
B(t, T) \equiv \frac{1}{M_{t}} E_{t}\left[M_{T}\right] .
$$

We also introduce the average discount rate ("yield") $Y(t, T)$ between time $t$ and time $T$ defined by

$$
Y(t, T) \equiv-\frac{1}{T-t} \log B(t, T) .
$$

In order to deal with asymptotic issues, we recall the following terminology. We say ${ }^{8}$ that two processes $X_{t}$ and $Y_{t}$ are asymptotically equivalent if $\lim _{t \rightarrow \infty} \frac{X_{t}}{Y_{t}}=1$, which we denote by $X_{t} \sim Y_{t}$. We say that a process $X_{t}$ asymptotically dominates a process $Y_{t}$ if $\lim _{t \rightarrow \infty} \frac{Y_{t}}{X_{t}}=0$.

The quantity $\frac{c_{i t}}{D_{t}}$ represents the consumption share of agent $i$ at time $t$ (in equilibrium). We also introduce the quantity

$$
\omega_{i t} \equiv \frac{b_{i} c_{i t}}{\sum_{j=1}^{N} b_{j} c_{j t}}
$$

\footnotetext{
${ }^{7}$ We refer to Duffie and Huang (1985) and to Riedel (2001) to show that our ArrowDebreu equilibrium can be implemented by continuous trading of such long-lived securities.

${ }^{8}$ As in e.g. Kogan et al. (2006).
} 
which represents the relative level of absolute risk tolerance ${ }^{9}$ of agent $i$ at time $t$, and plays an important part in describing the equilibrium (see, e.g. Jouini and Napp, 2007).

\section{Equilibrium in Homogeneous Economies}

We start by considering the equilibrium characteristics that would prevail in an economy made of agent $i$ only or that would prevail in our economy if all the initial endowment were concentrated on agent $i$.

We denote by $M_{i}$ the equilibrium state price density in an economy with only agent $i$. By the first order conditions in the homogeneous economies, we have

$$
M_{i t}=e^{-\rho_{i} t} Z_{i t} D_{t}^{-\gamma_{i}}=e^{-\left(\rho_{i}+\gamma_{i}\left(\mu-\frac{\sigma^{2}}{2}\right)+\frac{1}{2} \delta_{i}^{2}\right) t+\left(\delta_{i}-\gamma_{i} \sigma\right) W_{t}} .
$$

The market price of risk $\theta_{i} \equiv \frac{\mu_{S}(t)+D_{t} S_{t}^{-1}-r_{i t}}{\sigma_{S}(t)}$, the risk free rate $r_{i}$ and the survival index $\kappa_{i}$ (Yan, 2008) are respectively given ${ }^{10}$ by

$$
\begin{aligned}
\theta_{i} & =\left(\gamma_{i} \sigma-\delta_{i}\right), r_{i}=\rho_{i}+\gamma_{i} \mu_{i}-\frac{1}{2} \gamma_{i}\left(\gamma_{i}+1\right) \sigma^{2} \text { and } \\
\kappa_{i} & \equiv \rho_{i}+\gamma_{i}\left(\mu-\frac{\sigma^{2}}{2}\right)+\frac{1}{2} \delta_{i}^{2} .
\end{aligned}
$$

The risk free rate represents the agent's savings motives. The savings motives increase with pessimism and with patience. We index by $I_{0}$ the agent with the highest savings motives, i.e., such that $r_{I_{0}} \equiv \inf _{i} r_{i}$.

The market price of risk represents the agent's motives to invest in the risky asset. It increases with pessimism and with risk aversion. We index by $I_{\theta_{\max }}\left(I_{\theta_{\min }}\right)$ the agent with the highest (lowest) market price of risk.

The survival index satisfies $\kappa_{i}=-\frac{1}{t} E\left[\log M_{i t}\right]$ and can then be interpreted as the growth rate of the state price density $M_{i}$. It decreases with patience, rationality, and risk aversion when $\mu \geq \frac{\sigma^{2}}{2}$. The survival index differs from the risk free rate by an Itô's term, more precisely we have $r_{i}=\kappa_{i}-\frac{1}{2} \theta_{i}^{2}$. We index by $I_{K}$ the agent with the lowest survival index.

\footnotetext{
${ }^{9}$ The relative level of absolute risk tolerance of agent $i$ at time $t$ is given by $-\frac{u_{i}^{\prime}}{u_{i}^{\prime \prime}}\left(c_{i t}\right)\left[\sum_{j=1}^{N}-\frac{u_{i}^{\prime}}{u_{i}^{\prime \prime}}\left(c_{i t}\right)\right]^{-1}$.

${ }^{10}$ Letting $\mu_{M_{i}}$ and $\sigma_{M_{i}}$ respectively denote the drift and volatility of the state price density $M_{i}$, the market price of risk and the risk free rate satisfy $r_{i t}=-\mu_{M_{i}}(t)$ and $\theta_{i t}=-\sigma_{M_{i}}(t)$.
} 
We make the assumption that each of the criteria is minimal (or maximal) for one agent only, i.e., that $I_{0}, I_{K}, I_{\theta_{\min }}$ and $I_{\theta_{\max }}$ are well defined and unique. If there is only heterogeneity in time preference rates, the agent with the lowest survival index is also the agent with the highest savings motives (agent $I_{K}$ coincides with agent $I_{0}$ ) and is the most patient agent. If there is only heterogeneity in beliefs, agent $I_{K}$ is the most rational agent and differs from the agent with the highest savings motives who is the most pessimistic agent. If there is only heterogeneity in risk aversion and if $\mu>\frac{\sigma^{2}}{2}$, agent $I_{K}$ is the least risk averse agent.

The next proposition sums up the main results about the equilibrium characteristics in the homogeneous economies.

Proposition 3.1 In the homogeneous economies made of agent $i$ only, the following properties hold.

- The risk free rate $r_{i}$ and the market price of risk $\theta_{i}$ are constant and given by $\theta_{i}=\left(\gamma_{i} \sigma-\delta_{i}\right)$ and $r_{i}=\rho_{i}+\gamma_{i} \mu_{i}-\frac{1}{2} \gamma_{i}\left(\gamma_{i}+1\right) \sigma^{2}$. The stock's drift and volatility are also constant and given by $\sigma_{i S}=\sigma$ and $\mu_{i S}=$ $r_{i}+\sigma \theta_{i}$.

- The state price density $M_{i}$ can be written in the form $M_{i t}=e^{-\kappa_{i} t-\theta_{i} W_{t}}$ where $\kappa_{i} \equiv \rho_{i}+\gamma_{i}\left(\mu-\frac{\sigma^{2}}{2}\right)+\frac{1}{2} \delta_{i}^{2}$ denotes agent $i$ 's survival index. The state price density $M_{i t}$ is lognormal with average $e^{-r_{i} t}$ and median $e^{-\kappa_{i} t}$.

- When $\theta_{i}$ is nonnegative (resp. nonpositive), then the state price density $M_{i}\left(t, W_{t}\right)$ is decreasing (resp. increasing) in $W_{t}$, ranging from infinity to 0 , with $M_{i}(t, 0)=e^{-\kappa_{i} t}$.

- The state price density of the agent with the lowest (resp. highest) market price of risk dominates the other state price densities for positive

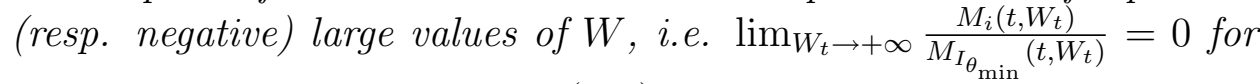
all $i \neq I_{\theta_{\min }}$ and $\lim _{W_{t} \rightarrow-\infty} \frac{M_{i}\left(t, W_{t}\right)}{M_{I_{\max }}\left(t, W_{t}\right)}=0$ for all $i \neq I_{\theta_{\max }}$.

- The state price density of the agent with the lowest survival index asymptotically dominates the other state price densities, i.e., $\lim _{t \rightarrow \infty} \frac{M_{i t}}{M_{I_{K}}}=0$ for all $i \neq I_{K}$

- The savings motives drive the risk free rate and the bond price. We have, for all $(t, T), B_{i}(t, T)=e^{-r_{i}(T-t)}$ and $Y_{i}(t, T)=r_{i}$. The bond 
price of the agent with the highest savings motives asymptotically dominates the other bond prices, i.e., $\lim _{T \rightarrow+\infty} \frac{B_{i}(t, T)}{B_{I_{0}}(t, T)}=0$ for all $i \neq I_{0}$.

In particular, different agents dominate different prices; agent $I_{0}$ dominates the asymptotic bond prices, agent $I_{K}$ asymptotically dominates ArrowDebreu prices, agent $I_{\theta_{\min }}$ dominates the prices of the Arrow-Debreu assets associated to very good states of the world and agent $I_{\theta_{\max }}$ dominates the prices of the Arrow-Debreu assets associated to very bad states of the world.

\section{Equilibrium state price density}

In the setting with heterogeneous agents, the first-order conditions of the agents optimization programs $\left(O_{i M}\right)$ give us the existence of Lagrange multipliers $\lambda_{i}$ such that, for all $i$, the equilibrium state price density is given by

$$
M_{t}=\lambda_{i} e^{-\rho_{i} t} Z_{i t} c_{i t}^{-\gamma_{i}} .
$$

Since prices are in terms of date 0 consumption goods, we have $M_{0}=1$ and $\lambda_{i}=c_{i 0}^{\gamma_{i}}$. The market clearing condition then leads to $\sum_{i=1}^{N} c_{i 0} e^{-\rho_{i} b_{i} t} Z_{i t}^{b_{i}} M_{t}^{-b_{i}}=$ $D_{t}$, which can be written in the form

$$
\sum_{i=1}^{N} c_{i 0}\left(\frac{M_{i}}{M}\right)^{b_{i}}=1
$$

When the agents have the same level of risk tolerance $b$ (and possibly differ in their beliefs or in their time preference rates), we get that the equilibrium state price density is a weighted power average of the state price densities in homogeneous economies (the power being given by the common level of risk tolerance). Indeed, we then easily deduce from equation (2) that $M=$ $\left(\sum_{i=1}^{N} c_{i 0} M_{i t}^{b}\right)^{1 / b}$. In our setting, the following lemma provides an expression of the state price density $M$ as well as bounds on $M$ in terms of the state price densities in the homogeneous economies. It is a direct analog of Lemma 2.1 from Cvitanić and Malamud (2008).

Lemma 4.1 1. Letting $F\left(a_{1}, \ldots, a_{n}\right)$ be the function defined as the unique solution to $\sum_{i=1}^{N} F^{-b_{i}} a_{i}^{b_{i}}=1$, we have $M=F\left(c_{10}^{\gamma_{1}} M_{1}, \ldots, c_{N 0}^{\gamma_{N}} M_{N}\right)$. 
2. Let $\Gamma \geq 1$ be such that $\Gamma b_{i}>1$ for all $i$ and $\gamma \leq 1$ be such that $\gamma b_{i} \leq 1$ for all $i$. Then,

$$
\left(\sum_{i=1}^{N} c_{i 0}^{\gamma_{i} / \gamma} M_{i}^{1 / \gamma}\right)^{\gamma} \leq M \leq\left(\sum_{i=1}^{N} c_{i 0}^{\gamma_{i} / \Gamma} M_{i}^{1 / \Gamma}\right)^{\Gamma}
$$

Notice that, up to a normalization, this means that the state price density $M$ is bounded below by a $\gamma$-average of the $M_{i}$ 's and is bounded above by a $\Gamma$ - average of the $M_{i}$ 's.

The state price density $M$ fluctuates in time and state of the world. We easily get from equation (2) that

$$
\min _{1 \leq i \leq N} M_{i} \leq M \leq \max _{1 \leq i \leq N} M_{i}
$$

For each state of the world, the state price density $M$ lies in the range bounded by the lowest and the highest state price densities and $M$ can then be interpreted as a kind of average of the state price densities $M_{i}$. The fact that the state price density $M$ is bounded by two "averages" of the individual state price densities (Lemma 4.1) and the properties of the state price densities obtained in Proposition 3.1 enable us to show how the state price density $M$ fluctuates with $W_{t}$ (or equivalently with aggregate endowment) as well as its asymptotic behavior.

Corollary 4.1 - The asymptotic behavior of the state price density is given by $M_{t} \sim c_{I_{K} 0}^{\gamma_{I_{K}}} M_{I_{K} t}$.

- If all the state price densities $M_{i t}=M_{i}\left(t, W_{t}\right)$ are decreasing in $W_{t}$, then

- the state price density $M_{t}=M\left(t, W_{t}\right)$ is also decreasing in $W_{t}$,

- the state price density $M_{t}=M\left(t, W_{t}\right)$ satisfies

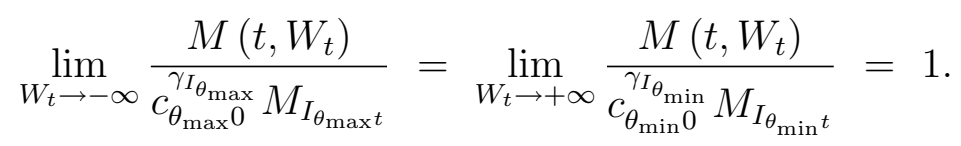

Asymptotically and in extreme states of the world, the state price density $M$ is equivalent to the state price density that would prevail in an economy made of homogeneous agents with a different endowment distribution. This 
class of homogeneous agents is given by the agent who dominates the individual state price density $M_{i}$ in the considered states of the world: agent $I_{K}$ asymptotically, agent $I_{\theta_{\max }}$ in the very bad states and agent $I_{\theta_{\min }}$ in the very good states.

The asymptotic result implies, in particular, that except agent $I_{K}$, all the agents have no price impact in the sense of Kogan et al. (2008, Definition 2) since we have for all $s>0$,

$$
\lim _{t \rightarrow \infty} \frac{M_{t+s} / M_{t}}{M_{I_{K} t+s} / M_{I_{K} t}}=1
$$

Later below, we shall analyze if the other agents really have no price impact in the sense that the prices of all assets are asymptotically the same as in the economy with the agent with the lowest survival index only.

In Section 6 we shall analyze the expression of the drift and volatility of the state price density $M$ (in other words the risk free rate and the market price of risk) and how they relate to the drift and volatility of $M_{I_{K}}, M_{I_{\theta_{\max }}}$ and $M_{I_{\theta_{\min }}}$ in extreme states of the world and asymptotically.

\section{Consumption shares and relative levels of risk tolerance}

In this section we analyze the expression and the behavior of the consumption shares and of the relative levels of risk tolerance. This analysis is interesting for survival issues, or more generally to know which agent dominates the market in terms of endowment shares. It will also prove to be useful to analyze the behavior of equilibrium characteristics, because, as we shall see in the next section, they rely on the relative levels of risk tolerance.

For each agent $i$, her consumption share at time $t$ is given by $\frac{c_{i t}}{D_{t}}$. We get from equation (1) that $\frac{c_{i t}}{D_{t}}=e^{-\rho_{i} b_{i} t} M_{t}^{-b_{i}} Z_{i t}^{b_{i}} c_{i 0}$, hence, using the expression of the state price densities $M_{i}$, we have

$$
\frac{c_{i t}}{D_{t}}=c_{i 0}\left(\frac{M_{i t}}{M_{t}}\right)^{b_{i}} .
$$

The consumption share can equivalently be written in the form

$$
\frac{c_{i t}}{D_{t}}=c_{i 0} M_{i}^{b_{i}} F^{-b_{i}}\left(c_{10}^{\gamma_{1}} M_{1}, \ldots, c_{N 0}^{\gamma_{N}} M_{N}\right) .
$$


The relative level of risk tolerance is given by $\omega_{i t} \equiv \frac{b_{i} c_{i t}}{\sum_{j=1}^{N} b_{j} c_{j t}}$. Differentiating the equation $\sum_{i=1}^{N} F^{-b_{i}} a_{i}^{b_{i}}=1$, we get $F_{a_{i}}\left(c_{10}^{\gamma_{1}} M_{1}, \ldots, c_{N 0}^{\gamma_{N}} M_{N}\right)=\frac{M_{t}}{c_{i 0}^{\gamma_{i}} M_{i}} \omega_{i t}$ hence the relative level of risk tolerance can be written in the form

$$
\omega_{i t}=c_{i 0}^{\gamma_{i}} M_{i} F^{-1} F_{a_{i}} .
$$

As in Kogan et al. (2006) or Yan (2008), we say that investor $i$ becomes extinct if $\lim _{t \rightarrow+\infty} \frac{c_{i t}}{D_{t}}=0$, that she survives if extinction does not occur and that she dominates the market asymptotically if $\lim _{t \rightarrow+\infty} \frac{c_{i t}}{D_{t}}=1$. We easily deduce from the properties of the state price densities obtained in Proposition 3.1 the following properties of the consumption shares and relative levels of risk tolerance, the first of which was obtained by Yan (2008).

Corollary 5.1 • Only the agent with the lowest survival index survives and dominates the market asymptotically, i.e., $\lim _{t \rightarrow \infty} \frac{c_{i t}}{D_{t}}=0$ for all $i \neq I_{K}$, and $\lim _{t \rightarrow \infty} \frac{c_{I_{K} t}}{D_{t}}=1$.

- Only the agent with the lowest survival index impacts asymptotically the relative level of risk tolerance, i.e., $\lim _{t \rightarrow \infty} \omega_{i t}=0$ for all $i \neq I_{K}$, and $\lim _{t \rightarrow \infty} \omega_{I_{K} t}=1$.

- We have $\lim _{W_{t} \rightarrow \infty} \omega_{i}\left(t, W_{t}\right)=\lim _{W_{t} \rightarrow \infty} \frac{c_{i}}{D}\left(t, W_{t}\right)=0$ for all $i \neq$ $I_{\theta_{\min }}$ and $\lim _{W_{t} \rightarrow \infty} \omega_{I_{\theta_{\min }}}\left(t, W_{t}\right)=\lim _{W_{t} \rightarrow \infty} \frac{{ }^{c_{I_{\min }}}}{D}\left(t, W_{t}\right)=1$. We have $\lim _{W_{t} \rightarrow-\infty} \omega_{i}\left(t, W_{t}\right) \stackrel{=}{=} \lim _{W_{t} \rightarrow-\infty} \frac{c_{i}}{D}\left(t, W_{t}\right)=0$ for all $i \neq I_{\theta_{\max }}$ and $\lim _{W_{t} \rightarrow-\infty} \omega_{I_{\theta_{\max }}}\left(t, W_{t}\right)=\lim _{W_{t} \rightarrow-\infty} \frac{c_{I_{\max }}}{D}\left(t, W_{t}\right)=1$.

- We have $\frac{\partial \omega_{i}\left(t, W_{t}\right)}{\partial W_{t}}=\omega_{i t}\left[b_{i}\left(\theta_{t}-\theta_{i}\right)-\sum_{j} \omega_{j t} b_{j}\left(\theta_{t}-\theta_{j}\right)\right]$ and there is a shift following good news in the relative levels of risk tolerance towards agents with a relatively high $b_{i}\left(\theta_{t}-\theta_{i}\right)$.

This implies that only the agent with the lowest survival index (resp. with the highest/lowest market price of risk) dominates the market in the sense of the consumption shares or in the sense of the risk tolerance asymptotically (resp. in very bad/good states of the world). As previously seen, this agent is the agent who values the wealth more than the other agents in the considered state.

Note that the agent with the highest $b_{i}\left(\theta_{t}-\theta_{i}\right)$ is the most optimistic agent when there is only heterogeneity in beliefs, and is the least risk averse 
agent when there is only heterogeneity in risk aversion levels. In both cases this agent is the one who has the highest risk exposure and is then the most favoured by good news.

\section{Risk free rate and market price of risk}

If we denote by $\mu_{M}$ and $\sigma_{M}$ the drift and the volatility of the state price density process $M$, it is easy to obtain as in the standard setting that the short term rate $r_{t}$ and the market price of risk $\theta_{t} \equiv \frac{\mu_{S}(t)+D_{t} S_{t}^{-1}-r_{t}}{\sigma_{S}(t)}$ are respectively given by $r_{t}=-\mu_{M}(t)$ and $\theta_{t}=-\sigma_{M}(t)$.

The next proposition gives us the expression of the risk free rate and of the market price of risk in our heterogeneous setting. Detemple and Murthy (1997, Proposition 5) gives the expression of the risk free rate and of the market price of risk in a model with portfolio constraints, heterogeneous beliefs, homogeneous risk aversion levels and homogeneous time preference rates.

Proposition 6.1 The risk free rate is given by

$$
\begin{aligned}
r_{t} & =\sum_{i=1}^{N} \omega_{i t} r_{i}-\left(\sum_{i=1}^{N} \theta_{i} \omega_{i t}\right)\left(\sum_{j=1}^{N} \theta_{j}\left(1-b_{j}\right) \omega_{j t}\right) \\
& +\frac{1}{2}\left(\sum_{k=1}^{N}\left(1-b_{k}\right) \omega_{k t}\right)\left(\sum_{i=1}^{N} \theta_{i} \omega_{i t}\right)^{2}+\frac{1}{2} \sum_{i=1}^{N}\left(1-b_{i}\right) \theta_{i}^{2} \omega_{i t}
\end{aligned}
$$

and the market price of risk is given by

$$
\theta_{t}=\sum_{i=1}^{N} \omega_{i t} \theta_{i}
$$

where we recall that $\omega_{j t} \equiv \frac{b_{j} c_{j t}}{\sum_{k} b_{k} c_{k t}}$, and where $\theta_{i} \equiv \gamma_{i} \sigma-\delta_{i}$ and $r_{i} \equiv \rho_{i}+$ $\gamma_{i} \mu_{i}-\frac{1}{2} \gamma_{i}\left(\gamma_{i}+1\right) \sigma^{2}$ respectively denote the market price of risk and the risk free rate in the economy populated by agent $i$ only.

The risk free rate and the market price of risk fluctuate in time and state of the world and these fluctutations are directly related to the fluctuations of the relative levels of risk tolerance $\omega_{i t}$. In particular, when one agent 
dominates the others in terms of risk tolerance then her individual market price of risk (risk free rate) dominates the equilibrium market price of risk (risk free rate). The market price of risk is a weighted average of the market prices of risk in the homogeneous economies. It fluctuates between the two bounds which are the lowest and the highest market price of risk in the different homogeneous economies. The risk free rate differs from an average of the homogeneous risk free rates and in particular can be lower than the lowest risk free rate or higher than the highest risk free rate ${ }^{11}$. More precisely, we get the following result.

Corollary 6.1 • The market price of risk satisfies

$$
\min _{i} \theta_{i} \leq \theta_{t} \leq \max _{i} \theta_{i}
$$

In addition, we have

$$
\lim _{W_{t} \rightarrow+\infty} \theta\left(t, W_{t}\right)=\min _{i} \theta_{i}=\theta_{I_{\theta_{\min }}}, \lim _{W_{t} \rightarrow-\infty} \theta\left(t, W_{t}\right)=\max _{i} \theta_{i}=\theta_{I_{\theta_{\max }}}
$$

and the asymptotic market price of risk is given by $\lim _{t \rightarrow \infty} \theta_{t}=\theta_{I_{K}}$.

- The risk free rate satisfies

$$
\begin{array}{ll}
r_{t} \leq \sum_{i=1}^{N} \omega_{i t} r_{i} \leq \max _{i} r_{i} & \text { if } \gamma_{i} \leq 1 \text { for all } i \\
r_{t} \geq \sum_{i=1}^{N} \omega_{i t} r_{i} \geq \min _{i} r_{i} \equiv r_{I_{0}} & \text { if } \gamma_{i} \geq 1 \text { for all } i .
\end{array}
$$

In addition, we have

$$
\lim _{W_{t} \rightarrow+\infty} r\left(t, W_{t}\right)=r_{I_{\theta_{\min }}}, \lim _{W_{t} \rightarrow-\infty} r\left(t, W_{t}\right)=r_{I_{\theta_{\max }}}
$$

and the asymptotic risk free rate is given by $\lim _{t \rightarrow \infty} r_{t}=r_{I_{K}}$.

\footnotetext{
${ }^{11}$ For instance, consider the case where only $\delta_{i}$ is heterogeneous. It is easy to see that $r_{t}$ is then given by

$$
r_{t}=E^{\omega_{t}}\left[r_{i}\right]+\frac{1}{2}(1-b) \operatorname{Var}^{\omega_{t}}\left[\delta_{i}\right]
$$

where $E^{\omega_{t}}$ and $V a r^{\omega_{t}}$ are respectively the expectation and the variance operators associated with the weights $\omega_{i t}$. In particular, in the case $N=2, e^{*^{1}}=e^{*^{2}}$ and $\delta_{1}=-\delta_{2}, r_{0}$ lies in $\left[r_{1}, r_{2}\right]$ if and only if $|1-b| \delta \leq 2 \gamma \sigma$.
} 
The result on the asymptotic risk free rate can be seen as the generalization of Yan (2008, Corollary 1) to the case with heterogeneous risk aversions and time preference rates. The asymptotic behavior of the risk free rate and of the market price of risk is driven by the agent with the lowest survival index only. She is the only surviving agent (in the sense of the consumption share or of the relative level of risk tolerance), hence is the only one to impact asymptotically the instantaneous risk free rate and market price of risk.

Analogously, only the agent with the lowest (resp. highest) market price of risk impacts the behavior of the risk free rate and of the market price of risk in the heterogeneous economy for very high (resp. very low) values of $W_{t}$. She is the only agent present in the economy (in the sense of the consumption shares or of the relative levels of risk tolerance) in those states. This is to be related to the results of Corollaries 4.1 and 5.1. In particular, the market price of risk in the heterogeneous economy reaches the two bounds in very good and very bad states of the world. It is minimal in very good states of the world, and maximal in very bad states of the world. The next corollary shows how the market price of risk fluctuates with aggregate endowment.

Corollary 6.2 The market price of risk $\theta_{t}=\theta\left(t, W_{t}\right)$ is monotone decreasing in $W_{t}$ for any parameters of the model. The market price of risk is always countercyclical.

Note that this countercyclicality property is consistent with the observed variations of the equity premium. Indeed, there is evidence that the equity premium is time varying and as noted by, e.g., Campbell and Cochrane (1999) "equity risk premia seem to be higher at business cycles troughs than they are at peaks". This result generalizes the result obtained by Jouini and Napp (2009) in the specific setting of agents who only differ in their beliefs and who are on average rational. It is quite striking to obtain the countercyclicality result for any distribution of the characteristics (risk aversion level, beliefs, time preference rates). It is heterogeneity and its impact on the fluctuations of the relative levels of risk tolerance $\omega_{i t}$ which generates this countercyclical behavior.

We also get monotonicity results for the risk free rate in the case of homogeneous risk aversion.

Corollary 6.3 If risk aversion is homogeneous, that is $b_{i}=b$ for all $i$, then

- if the sequences $-\sigma\left(\delta_{i}\right)+0.5(b-1)\left(\delta_{i}^{2}\right)-\rho_{i}$ and $\left(\delta_{i}\right)$ are anti-comonotone, then $r_{t}$ is procyclical 
- if the sequences $-\sigma\left(\delta_{i}\right)+0.5(b-1)\left(\delta_{i}^{2}\right)-\rho_{i}$ and $\left(\delta_{i}\right)$ are comonotone, then $r_{t}$ is countercyclical.

For instance, if time preference parameters are also homogeneous, and if agents hove logarithmic utility functions, we immediately get that the risk free rate is procyclical. For general utility functions, we still obtain the procyclicality result as long as agents are not biased in their beliefs. These results remain valid if time preference rates $\rho_{i}$ are no longer homogeneous but comonotone with the beliefs $\delta_{i}$. These results are consistent with observed behavior since empirical studies have confirmed that the short term rate is a procyclical indicator of economic activity (see e.g. Friedman, 1986, Blanchard and Watson, 1986).

\section{Bond Prices}

As seen in Section 3, in the homogeneous economies, the average discount rate between time $t$ and $T$ is the same for all $(t, T)$ and given by the constant risk free rate. Indeed, we have in the homogeneous economy made of agent $i$ only, $B_{i}(t, T)=e^{-(T-t) r_{i}}$, and $Y_{i}(t, T)=r_{i}$. The yield curves, representing, for all time $t$, the discount rates $Y_{i}(t, T)$ as a function of $T-t$, are the same for all time $t$ and flat.

In the heterogeneous economy, the yield curves are not flat. The instantaneous discount rate defined by $\lim _{T \rightarrow t} Y(t, T)$ is given by the risk free rate $r_{t}$. The next proposition characterizes the asymptotic discount rate.

Proposition 7.1 The asymptotic average discount rate is determined by the agent with the highest savings motives, i.e., for all t,

$$
\lim _{T \rightarrow+\infty} Y(t, T)=r_{I_{0}} .
$$

The same reasoning as above holds: when one agent dominates the individual price of an asset then she makes the price of that asset in the heterogeneous economy. As seen in Proposition 3.1, the agent with the highest savings motives dominates the price of the very long term bond because it is most attractive for her. The agent with the highest savings motives then drives the asymptotic average discount rate. This proposition is the extension to the setting with three possible sources of heterogeneity of the proposition of Gollier and Zeckhauser (1996) for the case of heterogeneous time preference 
rates, of Wang (1996) for heterogeneous levels of risk aversion and of Jouini et al. (2008) for heterogeneous beliefs.

In the setting with heterogeneous time preference rates only, the same agent drives the asymptotic discount rate and the asymptotic risk free rate. Indeed, in that case, the agent with the lowest survival index is also the agent with the highest savings motives, namely the most patient agent. Apart from this setting, it is quite striking that the agent that drives the asymptotic average discount rate differs from the agent that drives the asymptotic risk free rate, even though the discount rate is an average of the risk free rates. Indeed, we have $Y(t, T)=-\frac{1}{T-t} \log E_{t}^{Q}\left[\exp -\int_{t}^{T} r_{s} d s\right]$ where $Q$ is the risk-neutral probability measure, with $r_{s} \rightarrow r_{I_{K}}$ and $Y(t, T) \rightarrow_{T \rightarrow \infty} r_{I_{0}}$. Analogously, we have $Y(t, T)=-\frac{1}{T-t} \log E_{t}\left[\frac{M_{T}}{M_{t}}\right]$ with $\frac{M_{T}}{M_{t}} \sim \frac{M_{I_{K} T}}{M_{I_{K}}}$ and $Y(t, T) \sim Y_{I_{0}}(t, T)$.

In the case with heterogeneous beliefs only, the risk free rate converges to the rate of the most rational agent whereas the asymptotic discount rate is driven by the most pessimistic agent.

In particular, Proposition 7.1 as well as Corollary 6.1 imply that when $t$ is large enough, the yield curve representing $Y(t, T)$ as a function of $(T-t)$ is driven by the risk free rate of the agent with the lowest survival index (agent $\left.I_{K}\right)$ at one end of the yield curve, i.e., for small values of $(T-t)$, whereas at the other end, i.e., for $(T-t)$ large enough, it is driven by the risk free rate of the agent with the highest savings motives or equivalently the lowest risk free rate (agent $I_{0}$ ). The aim of the remainder of this section is to show that the yield curve is defined stepwise, and that each subinterval is associated with a given agent in the sense that the marginal rate on that subinterval corresponds to the rate in the economy made of that agent only. Moreover, that agent is the agent who values the most a given zero coupon bond associated to the subinterval and is characterized by a maximization program involving a weighted average of the savings motives and of the survival index.

In the homogeneous economy made of agent $i$ only, the price, seen from date 0 , of a zero coupon bond between time $t$ and time $T$ and in state $\omega$ is given by $E_{t}\left[M_{i T}\right]=e^{-r_{i}(T-t)} e^{-\kappa_{i} t-\theta_{i} W_{t}}$. This implies that for $\lambda \in[0,1]$, we have $E_{\lambda T}\left[M_{i T}\right]=e^{-l_{i}(\lambda) T} e^{-\theta_{i} W_{\lambda T}}$ where

$$
l_{i}(\lambda)=\left[\lambda \kappa_{i}+(1-\lambda) r_{i}\right]=\left[\kappa_{i}-(1-\lambda) \frac{1}{2} \theta_{i}^{2}\right]
$$

is a weighted average of the survival index and of the risk free rate. Since $\left(l_{i}(\lambda), \lambda \in[0,1]\right)$ is a family of line segments, there exist pairs of values 
$\left(\left(I_{j}, \lambda_{j}\right), j=1, \cdots, K\right)$ such that

$$
\min _{i} l_{i}(\lambda)=l_{I_{j}}(\lambda) \text { for all } \lambda \in\left(\lambda_{j}, \lambda_{j+1}\right)
$$

where $\lambda_{0}=0$ and $\lambda_{K+1}=1$. For $\lambda$ near 0 , agent $I_{0}$ satisfies $r_{I_{0}}=\inf _{i} r_{i}$ and for $\lambda$ near 1 , agent $I_{K}$ satisfies $\kappa_{I_{K}}=\inf _{i} \kappa_{i}$.

The index $l_{i}(\lambda)$ drives the asymptotic behavior of the price $E_{\lambda T}\left[M_{i T}\right]$ in the sense that $\lim _{T \rightarrow \infty} \frac{E_{\lambda T}\left[M_{i T}\right]}{E_{\lambda T}\left[M_{I_{j} T}\right]}=0$ for all $i \neq I_{j}$ when $\lambda \in\left(\lambda_{j}, \lambda_{j+1}\right)$. This is due to the fact that the price $E_{t}\left[M_{i T}\right]$ involves both the state price density $M_{i t}$ whose asymptotic behavior is driven by the survival index and the bond price $B_{i}(t, T)$, whose asymptotic behavior is driven by the savings motives. For $\lambda=0$, we retrieve the fact that agent $I_{0}$ (with the lowest risk free rate) dominates the prices of the zero coupon bond $B_{i}(0, T)$ when $T$ is large enough. For $\lambda=1$, we retrieve the fact that agent $I_{K}$ (with the lowest survival index) dominates the state price densities $M_{i T}$ for $T$ large enough. For $\lambda \in(0,1)$, we obtain that agent $I_{j}$ (with the lowest index $l_{i}(\lambda)$, mixing the survival index and the savings motives) dominates the prices $E_{\lambda T}\left[M_{i T}\right]$ when $T$ is large enough.

Consider the case with heterogeneity in beliefs only. Then agent $I_{0}$ is the most pessimistic agent and agent $I_{K}$ is the most rational agent. Agent $I_{1}$ is the most pessimistic agent once agent $I_{0}$ is excluded, agent $I_{2}$ is the most pessimistic agent once agents $I_{0}$ and $I_{1}$ are excluded, etc. Moreover, the intervals $\left(\lambda_{j}, \lambda_{j+1}\right)$ on which $l_{I_{j}}(\lambda)=\min _{i} l_{i}(\lambda)$ are given by $\lambda_{j}=\frac{2 \gamma \sigma}{2 \gamma \sigma-\left(\delta_{I_{j-1}+\delta_{I_{j}}}\right)}$. Note that apart from agent $I_{K}$ (who might be optimistic or pessimistic) all the agents $I_{j}$ (for $j=0, \ldots, K-1$ ) are pessimistic. This is due to the following: In the case with heterogeneity on the beliefs only, minimizing $l_{i}(\lambda)$ amounts to minimizing the average of the survival index and of the risk free rate associated to the $i-t h$ agent. The survival index reaches its minimum for the lowest $\delta_{i}$ in absolute value (i.e., for the most rational agent), while the risk free rate increases with $\delta_{i}$. Starting from the most rational agent, it is clear that the only way to possibly decrease $l_{i}(\lambda)$ consists in moving in the direction of more pessimism.

In our heterogeneous economy, we obtain as a corollary to Equation (3) that

$$
\left(\sum_{i=1}^{N} c_{i 0}^{\gamma_{i} / \gamma} E_{t}\left[M_{i T}\right]^{1 / \gamma}\right)^{\gamma} \leq E_{t}\left[M_{T}\right] \leq\left(\sum_{i=1}^{N} c_{i 0}^{\gamma_{i} / \Gamma} E_{t}\left[M_{i T}\right]^{1 / \Gamma}\right)^{\Gamma}
$$


which leads to the following results on the bond prices.

Proposition 7.2 - The bond prices satisfy

$$
E_{t}\left[M_{\alpha t}\right] \sim c_{I_{j} 0}^{\gamma_{I_{j}}} E_{t}\left[M_{I_{j} \alpha t}\right] \text { and } B(t, \alpha t) \sim \frac{c_{I_{j} 0}^{\gamma_{I_{j}}}}{c_{I_{K} 0}^{\gamma_{K}}} \frac{E_{t}\left[M_{I_{j} \alpha t}\right]}{M_{I_{K} \alpha t}}
$$

for all $\alpha \in\left(\frac{1}{\lambda_{j+1}}, \frac{1}{\lambda_{j}}\right)$.

- We have, for $\alpha \in\left(\frac{1}{\lambda_{j}}, \frac{1}{\lambda_{j-1}}\right)$,

$$
Y(\alpha) \equiv \lim _{t \rightarrow \infty} Y(t, \alpha t)=\frac{1}{\alpha-1}\left[\kappa_{I_{K}}-\alpha l_{I_{j}}(1 / \alpha)\right]
$$

and the convergence is uniform on compact subsets of $(1, \infty)$. We have $\lim _{\alpha \rightarrow 1} Y(\alpha)=r_{I_{K}}$ and $\lim _{\alpha \rightarrow \infty} Y(\alpha)=r_{I_{0}}$.

- The marginal rates associated to the asymptotic yield curve (the instantaneous forward rates) are given by

$$
\frac{d}{d \alpha}[Y(\alpha)(\alpha-1)]=r_{I_{j}}
$$

on $\left(\frac{1}{\lambda_{j+1}}, \frac{1}{\lambda_{j}}\right)$.

The above result provides then the shape of the asymptotic yield curve. However, it is important to notice that, asymptotically, yield curves at different dates are obtained through homothetic transformations and not through translations. In other words, for $t$ large enough, all yield curves will have the same shape, but at different scales.

Different segments of the (asymptotic) yield curve are determined by different agents with different characteristics. More precisely, the marginal discount rate for the interval $\left(\frac{1}{\lambda_{j+1}}, \frac{1}{\lambda_{j}}\right)$ is determined by agent $I_{j}$. These results are particularly in line with the Preferred Habitat Theory (PHT) developed by Modigliani and Sutch (1966). Following this theory, if an investor's investment horizon coincides with the maturity of a zero-coupon bond then she will consider the long-term bond as riskless and require a premium for holding short-term bonds. Each investor has then a preferred habitat that corresponds to her "natural" investment horizon. This investment horizon 
might result from institutional factors, regulatory constraints or from different levels of risk exposure of the background portfolio at different maturities. In our framework, this investment horizon naturally emerges for each agent, as the result of agents' heterogeneity.

It is interesting to note that even though only one agent survives in the long term, non surviving agents might continue to have an impact on the yield curve. One may argue that the impact of agent $I_{j}$ is only between $\left(\frac{1}{\lambda_{j+1}} t, \frac{1}{\lambda_{j}} t\right)$ and is then at more and more distant horizons when $t$ increases. However, we can construct examples where non surviving agents impact prices and where this impact does not vanish asymptotically, as illustrated in the following example.

Example 7.1 Assuming heterogeneity in beliefs only, we know that $r_{I_{K}}$ corresponds to the risk free rate in the economy populated by the most rational agent and $r_{I_{0}}$ corresponds to the risk free rate in the economy populated by the most pessimistic agent only. Let us consider an asset (growing perpetuity) with a deterministic dividend flow $d_{t}=d_{0} \exp (\hat{r} t)$ with $r_{I_{0}}<\hat{r}<r_{I_{K}}$. The price at date $t$ of this asset in the economy populated by agent $I_{K}$ only is given by

$$
p_{t}=d_{0} \exp \left(r_{I_{K}} t\right) \int_{t}^{\infty} \exp \left(\left(\hat{r}-r_{I_{K}}\right) s\right) d s=\frac{d_{0}}{r_{I_{K}}-\hat{r}} \exp (\hat{r} t)
$$

in terms of date $t$ prices. On the other hand, the price $p_{t}^{\prime}$ of this asset in the heterogeneous economy is infinite in date $t$ prices terms. Indeed, if we denote by $\bar{r}_{s}$ the marginal discount rate (from date $t$ point of view) at date $s$ (i.e. $\left.\bar{r}_{s}=-\frac{1}{B(t, s)} \frac{\partial B(t, s)}{\partial s}\right)$ we know that $\bar{r}_{s}$ is arbitrarily close to $r_{I_{0}}$ for $s$ sufficiently large. More precisely, let s such that $\bar{r}_{v} \leq \hat{r}-\varepsilon$ for $\varepsilon>0$ and for all $v \geq s$. We have

$$
\begin{aligned}
p_{t}^{\prime} & =d_{0} \exp (\hat{r} t) \int_{t}^{\infty} \exp \left(\int_{t}^{u}\left(\hat{r}-\bar{r}_{v}\right) d v\right) d u \\
& \geq d_{0} \exp (\hat{r} t) \exp \left(\int_{t}^{s}\left(\hat{r}-\bar{r}_{v}\right) d v\right) \int_{s}^{\infty} \exp \left(\int_{s}^{u}\left(\hat{r}-\bar{r}_{v}\right) d v\right) d u
\end{aligned}
$$

and it is easy to see that the last integral is infinite and so is $p_{t}^{\prime}$.

In order to explore further the shape of the yield curve and the relative impact of the different agents, we consider the case of an economy populated 
by continuously many agents. Let us first assume that the characteristics $(\rho, \gamma, \delta)$ of these agents are continuously distributed on some subset $U$ of $\mathbb{R}_{+} \times \mathbb{R}_{+} \times \mathbb{R}$. In such a setting, each $\lambda$ is the preferred habitat of the agent whose characteristics $(\rho(\lambda), \gamma(\lambda), \delta(\lambda))$ satisfy

$(\rho(\lambda), \gamma(\lambda), \delta(\lambda))=\arg \max _{(\rho, \gamma, \delta) \in U}\left\{-\left(\rho+\gamma\left(\mu-\frac{1}{2} \sigma^{2}\right)+\frac{1}{2} \delta^{2}\right)+\frac{1}{2}(1-\lambda)(\delta-\gamma \sigma)^{2}\right\}$.

Agent $I_{K}$ corresponds to $\lambda=1$ and we get for the yield between $t$ and $T \geq t$ that

$$
\begin{aligned}
& Y(t, T) \\
& \sim(T-t)^{-1}\left(T \rho\left(\frac{t}{T}\right)-t \rho(1)-\left(t \gamma(1)-T \gamma\left(\frac{t}{T}\right)\right)\left(\mu-\frac{\sigma^{2}}{2}\right)\right. \\
& \left.-\frac{1}{2}\left(t \delta^{2}(1)-T \delta^{2}\left(\frac{t}{T}\right)\right)-\frac{T-t}{2}\left(\delta\left(\frac{t}{T}\right)-\gamma\left(\frac{t}{T}\right) \sigma\right)^{2}\right)
\end{aligned}
$$

where the equivalence is meant asymptotically in $t$, the ratio $t / T$ being fixed.

Example 7.2 Assume that there are continuously many beliefs $\delta$, while the discount rate $\rho$ and the risk aversion $\gamma$ are constant across the agents. More precisely, let us take $U=\{\rho\} \times\{\gamma\} \times\left[\delta_{\min }, \delta_{\max }\right]$. Then we get

$$
(\rho(\lambda), \gamma(\lambda), \delta(\lambda))=(\rho, \gamma, \gamma \sigma(1-1 / \lambda))
$$

as long as $\gamma \sigma(1-1 / \lambda)$ is in $\left[\delta_{\min }, \delta_{\max }\right]$. For $1 / \lambda$ large enough, we have $\delta(\lambda)=\delta_{\min }$. For the sake of simplicity, we assume that $0 \in\left[\delta_{\min }, \delta_{\max }\right]$ which gives us that $\delta(1)=0$. Substituting in equation (5), we get

$Y(t, T)$

$$
\begin{aligned}
& \sim \rho+\gamma\left(\mu-\frac{1}{2} \sigma^{2}\right)-\frac{1}{2} \gamma^{2} \sigma^{2} \frac{T}{t}, \quad \text { for } \frac{T}{t} \in\left[1,1-\frac{\delta_{\min }}{\gamma \sigma}\right] \\
& \sim \rho+\gamma\left(\mu-\frac{1}{2} \sigma^{2}\right)-\frac{1}{2}\left(\delta_{\min }-\gamma \sigma\right)^{2}+\frac{1}{2} \frac{T}{T-t} \delta_{\min }^{2}, \quad \text { for } \frac{T}{t} \in\left[1-\frac{\delta_{\min }}{\gamma \sigma}, \infty\right)
\end{aligned}
$$

The asymptotic term structure is inverted. It is linear until a certain threshold after which it converges asymptotically to the rate of the most pessimistic agent. As in the discrete setting, the different habitats are ordered on the time line by the level of pessimism of the associated agents. 
Example 7.3 In the case where only $\rho$ varies, i.e., $U=\left[\rho_{\min }, \rho_{\max }\right] \times\{\gamma\} \times$ $\{\delta\}$, we have

$$
(\rho(\lambda), \gamma(\lambda), \delta(\lambda))=\left(\rho_{\min }, \gamma, \delta\right)
$$

and the asymptotic term structure is constant. The whole asymptotic yield curve is associated to the lowest level of impatience.

Example 7.4 Consider now the case where only $\gamma$ varies. More precisely, suppose $U=\{\rho\} \times\left[\gamma_{\min }, \gamma_{\max }\right] \times\{\delta\}$. It is shown in the Appendix that for the case where the economy is shrinking, $\mu<\sigma^{2} / 2$, the whole yield curve (which is flat in this case) is associated to a single agent (the most riskaverse agent with $\gamma=\gamma_{\max }$, or the least risk-averse agent with $\gamma=\gamma_{\min }$, depending on how large is $\left.\gamma_{\max }\right)$. When the economy is growing, if the highest risk aversion is large enough, the yield curve is determined for short horizons by the agent with the lowest level of risk aversion (i.e., $\gamma=\gamma_{\min }$ ) and for long horizons by the agent with the highest level of risk aversion (i.e., $\gamma=$ $\left.\gamma_{\max }\right)$. We have then two different habitats and the more distant one in time is associated to a higher level of risk aversion than the less distant one. As noted by Wang (1996), long term bonds are more attractive to more risk averse agents as hedging instruments against future downturns of the economy. Indeed, the more risk averse investors are more averse to low levels of future consumption. Consequently they exert a stronger influence on their equilibrium price. However, $\gamma_{\max }$ should be large enough with respect to $\gamma_{\min }$, for this phenomenon to occur. If not, we may have an inversion: $\gamma_{\max }$ determines the short term rates and $\gamma_{\min }$ the long term ones.

\section{Stock price and cumulative returns}

We have determined the expression of the market price of risk $\theta_{t} \equiv \frac{\mu_{S}(t)+D_{t} S_{t}^{-1}-r_{t}}{\sigma_{S}(t)}$ and of the risk free rate $r_{t}$ and analyzed their asymptotic properties in Section 6. We now analyze the expression of the drift $\mu_{S}(t)$ and volatility $\sigma_{S}(t)$ of the stock price and their asymptotic properties. We also analyze the asymptotic properties of the price dividend ratio and of the cumulative returns. In particular, are they given by the quantities of the economy made of the surviving agent only?

We recall that in the homogeneous economies the volatility is a constant given by $\sigma$. In the homogeneous economy, populated only by agent $i$, it is easy to obtain that the stock price is finite if and only if, as in Yan (2008), 
$\rho_{i}+\left(\gamma_{i}-1\right) \mu_{i}-\frac{1}{2} \gamma_{i}\left(\gamma_{i}-1\right) \sigma^{2}>0$. The stock price-dividend ratio at time $t$ is then constant and given by

$$
\left(\frac{S}{D}\right)_{i} \equiv E_{t}\left[\int_{t}^{\infty} \frac{M_{i \tau} D_{\tau}}{M_{i t} D_{t}} d \tau\right]=\left[\rho_{i}+\left(\gamma_{i}-1\right) \mu_{i}-\frac{1}{2} \gamma_{i}\left(\gamma_{i}-1\right) \sigma^{2}\right]^{-1} .
$$

The cumulative expected return on rolling all the money in stock between time $t$ and time $T$ is then given by

$$
R_{i}(t, T) \equiv E_{t}\left[\frac{S_{i T}}{S_{i t}} e^{\int_{t}^{T} \frac{D_{\tau}}{S_{i \tau}} d \tau}\right]=e^{\left[\mu+\left[\left(\frac{S}{D}\right)_{i}\right]^{-1}\right](T-t)}
$$

and the associated yield curve,

$$
T \rightarrow \frac{1}{T-t} \log R_{i}(t, T)=\mu+\rho_{i}+\left(\gamma_{i}-1\right) \mu_{i}-\frac{1}{2} \gamma_{i}\left(\gamma_{i}-1\right) \sigma^{2}
$$

is flat and the same for all $t$.

\subsection{Volatility and price dividend ratio}

In our heterogeneous economy, we obtain the following results on the volatility and the price dividend ratio.

Recall that $\theta_{i}=\gamma_{i} \sigma-\delta_{i}$.

Proposition 8.1 1. The volatility parameter of the stock price is given by

$$
\sigma_{S}(t)=\sigma+\frac{E_{t}\left[\int_{t}^{\infty}\left(\theta_{t}-\theta_{\tau}\right) M_{\tau} D_{\tau} d \tau\right]}{E_{t}\left[\int_{t}^{\infty} M_{\tau} D_{\tau} d \tau\right]}
$$

In particular,

$$
\sigma+\min _{i} \theta_{i}-\max _{i} \theta_{i} \leq \sigma_{t}^{S} \leq \sigma+\max _{i} \theta_{i}-\min _{i} \theta_{i}
$$

2. The asymptotic stock price volatility satisfies

$$
\lim _{t \rightarrow \infty} \sigma_{S}(t)=\sigma
$$

3. The asymptotic price dividend ratio satisfies

$$
\lim _{t \rightarrow \infty} \frac{S_{t}}{D_{t}}=\left(\frac{S}{D}\right)_{I_{K}}
$$


4. Suppose risk aversion is homogeneous. Then,

- If $0.5 b(1-b) \delta_{i}^{2}+b \rho_{i}-b \sigma \delta_{i}$ is anti-co-monotone with $\delta_{i}$ then $\frac{S_{t}}{D_{t}}$ is procyclical (increases with $W_{t}$ ) and the excess volatility is positive, i.e. $\sigma_{S}(t) \geq \sigma$.

- If $0.5 b(1-b) \delta_{i}^{2}+b \rho_{i}-b \sigma \delta_{i}$ is co-monotone with $\delta_{i}$ then $\frac{S_{t}}{D_{t}}$ is countercyclical (decreases with $W_{t}$ ) and the excess volatility is negative, i.e. $\sigma_{S}(t) \leq \sigma$.

The volatility is not a constant as in the standard setting, due to the stochastic market price of risk. It can fluctuate in time and state of the world. The previous proposition gives us the range in which it fluctuates. As far as asymptotic properties are concerned, we obtain a positive answer to the question raised above. We obtain that only the surviving agent (i.e., the agent with the lowest survival index) has an impact on the asymptotic volatility and price dividend ratio.

However, as in the case of bonds, we now show that even though non surviving agents do not have an impact on the long run volatility and price dividend ratio, they may have an impact on the long run returns.

\subsection{Cumulative returns}

The cumulative expected return on rolling all the money in the stock between time $t$ and $T$ is given by

$$
\begin{aligned}
R(t, T) & =E_{t}\left[\frac{S_{T}}{S_{t}} e^{\int_{t}^{T} D_{s} S_{s}^{-1} d s}\right] \\
& =E_{t}^{(1)}\left[\frac{S_{T}}{D_{T}} e^{\int_{t}^{T} D_{s} S_{s}^{-1} d s}\right] E_{t}\left[D_{T}\right] \frac{D_{t}}{S_{t}}
\end{aligned}
$$

where $P_{T}^{(1)}$ is the probability measure on $\mathcal{F}_{T}$ whose density with respect to the restriction $P_{T}$ of $P$ on $\mathcal{F}_{T}$ is proportional to $D_{T}$. We also denote by $P^{(1)}$ the extension ${ }^{12}$ of the probability measures $P_{T}^{(1)}$ to the set of infinite paths.

Equation (9) shows that the asymptotic behavior of $\frac{S_{t}}{D_{t}}$ is as a key element in the determination of the asymptotic cumulative equity return. As seen in

\footnotetext{
${ }^{12}$ The existence of such a probability measure is guaranted by the Kolmogorov extension Theorem.
} 
Proposition 8.1, this ratio is asymptotically given by $\left(\frac{S}{D}\right)_{I_{K}}$ and is deterministic. However, even though this convergence is an almost sure convergence under $P$, it is not clear whether or not the limit remains the same ${ }^{13}$ under $P^{(1)}$. In fact, the optimal consumption of agent $i$ can be rewritten as follows

$$
c_{i t}=e^{-\rho_{i}^{(1)} b_{i} t} M_{t}^{-b_{i}}\left(Z_{i t}^{(1)}\right)^{b_{i}} c_{i 0}
$$

where

$$
\rho_{i}^{(1)}=\rho_{i}-\delta_{i} \sigma^{2} \quad \text { and } \quad Z_{i t}^{(1)}=e^{\delta_{i} W_{t}^{(1)}-\frac{1}{2} \delta_{i}^{2} t}
$$

and where $W_{t}^{(1)}$ is a standard Brownian motion under $P^{(1)}$. Thus, under this new measure everything looks the same, apart from the fact that agents have discount rates given by $\rho_{i}^{(1)}=\rho_{i}-\delta_{i} \sigma^{2}$ and that the drift is given by $\mu^{(1)}=\mu+\sigma^{2}$. This means that, under $P^{(1)}$, the surviving agent is no more agent $I_{K}$ but agent $A(1)$ characterized by

$$
\left(\rho_{A(1)}^{(1)}+\gamma_{A(1)}\left(\mu^{(1)}-\frac{1}{2} \sigma^{2}\right)+\frac{1}{2} \delta_{A(1)}^{2}\right)=\min _{i}\left(\rho_{i}^{(1)}+\gamma_{i}\left(\mu^{(1)}-\frac{1}{2} \sigma^{2}\right)+\frac{1}{2} \delta_{i}^{2}\right) .
$$

This suggest that survival and long run impact are different concepts. In the next we will illustrate the fact that the long run impact is determined by different agents depending on the asset under consideration.

Intuitively, one would expect from equation (9) that the cumulative equity returns converge to those determined by agent $A(1)$. In fact, the long run return in the homogeneous economy populated by agent $A(1)$ only provides a lower bound for the long run return in our economy. Since a change of probability leads to a change of surviving agent, it is possible to obtain other lower bounds by the introduction of well chosen artificial probabilities. The next proposition provides such a lower bound based on the consideration of a parametrized family of such artificial probabilities.

Proposition 8.2 Let $t=\lambda T$. We have

$$
\lim \inf _{T \rightarrow \infty}(T-t)^{-1} \log R(t, T) \geq \mu+\max _{\alpha}\left(-\frac{1}{2} \sigma^{2}(1-\alpha)^{2}+\left(\frac{S}{D}\right)_{A(\alpha)}^{-1}\right)
$$

\footnotetext{
${ }^{13}$ Indeed, the restrictions of the measures $P$ and $P^{(1)}$ on each sigma-algebra $F_{t}$ are equivalent, but they are not equivalent on $F_{\infty}$. This is why it is possible to get a different almost sure limit.
} 
where $A(\alpha)$ is characterized by

$$
\begin{aligned}
\rho_{A(\alpha)}-\delta_{A(\alpha)} \sigma^{2} \alpha+ & \gamma_{A(\alpha)}\left(\mu-\frac{1}{2} \sigma^{2}+\sigma^{2} \alpha\right)+\frac{1}{2} \delta_{A(\alpha)}^{2} \\
& =\min _{i}\left(\rho_{i}-\delta_{i} \sigma^{2} \alpha+\gamma_{i}\left(\mu-\frac{1}{2} \sigma^{2}+\sigma^{2} \alpha\right)+\frac{1}{2} \delta_{i}^{2}\right)
\end{aligned}
$$

and were $r^{(A(\alpha))}$ corresponds to the instantaneous riskless rate that would prevail in the economy populated by agent $A(\alpha)$.

Example 8.1 Assume that all agents have the same level of risk aversion $\gamma$ and the same time preference parameter $\rho$, but have heterogeneous beliefs that vary continuously taking values in $\left[\delta_{\min }, \delta_{\max }\right]$ with $\delta_{\min }<0$ and $\delta_{\max }>$ $[(\gamma-1) \sigma+1] \sigma^{2}>0$. We have

$$
\rho-\delta_{A(\alpha)} \sigma^{2} \alpha+0.5 \delta_{A(\alpha)}^{2}=\min _{i}\left(\rho-\delta_{i} \sigma^{2} \alpha+\frac{1}{2} \delta_{i}^{2}\right)
$$

which leads to

$$
\delta_{A(\alpha)}=\sigma^{2} \alpha
$$

as long as $\sigma^{2} \alpha \in\left[\delta_{\min }, \delta_{\max }\right]$. We have then

$$
\begin{gathered}
\lim \inf _{T \rightarrow \infty}(T-t)^{-1} \log R(t, T) \\
\geq \gamma \mu+\rho-0.5(\gamma-1) \sigma^{2}-(\gamma-1)^{2} \sigma^{2}+\sigma^{2} \max _{\alpha \in\left[\frac{\delta_{\min }}{\sigma^{2}}, \frac{\delta_{\max }}{\sigma^{2}}\right]}\left(-0.5(1-\alpha)^{2}+(\gamma-1) \sigma \alpha\right)
\end{gathered}
$$

The maximum is reached for

$$
\alpha^{*}=(\gamma-1) \sigma+1
$$

which gives

$$
\delta_{A^{*}}=\sigma^{2}((\gamma-1) \sigma+1)>0
$$

By construction, the long run return in this economy is higher than the long run return in the economy populated by agent $A^{*}$ only. Note also that

$$
\mu+\left(\frac{S}{D}\right)_{i}^{-1}=\mu+\rho+(\gamma-1)\left(\mu-0.5 \sigma^{2}+\sigma \delta_{i}+(1-\gamma) \sigma^{2}\right)
$$

which means that the long run return in the homogeneous economies increases with $\delta_{i}$ if only if $\gamma>1$. In this case we also have that the long run return in the 
homogeneous economy goes to infinity when $\delta_{i}$ goes to infinity. Consequently, for $\gamma>1$, we have that the long run return is higher than the long run return in the homogeneous economy populated by agent $A^{*}$ with $\delta_{A^{*}}=\sigma^{2}((\gamma-1) \sigma+$ 1) $>0$. The long run return in our economy corresponds then to the long return in an homogeneous economy populated by an agent $B$ with $\delta_{B} \geq \delta_{A}>0$ and such that

$\lim \inf _{T \rightarrow \infty}(T-t)^{-1} \log R(t, T)=\mu+\rho+(\gamma-1)\left(\mu-0.5 \sigma^{2}+\sigma \delta_{B}+(1-\gamma) \sigma^{2}\right)$.

In conclusion, in our economy we have that the long run return is determined by the agent with $\delta=\delta_{B}>0$ while the asymptotic long run discount rate is determined by the agent with $\delta=\delta_{\min }<0$ and the asymptotic short rate, volatility and stock price are determined by the agent with $\delta=0$ which is the only surviving agent.

\subsection{Optimal Portfolios}

Let us consider the investment strategy of agent $i$ in the risky and in the riskless aset that permits to implement the equilibrium consumption process $\left(c_{i t}\right)$. Such a strategy is characterized by a process $\pi_{i t}$ that corresponds to the number of shares of the risky asset held at date $t$ by the agent under consideration. The number of shares of riskless asset is determined by the dynamic budget constraint and if we denote by $w_{i t}$ the financial wealth of agent $i$ at date $t$ along this strategy, we have

$$
\begin{aligned}
d w_{i t} & =w_{i t}\left(r_{t} d t+\pi_{i t}\left(S_{t}^{-1}\left(d S_{t}+D_{t} d t\right)-r_{t} d t\right)\right)-c_{i t} d t \\
& =w_{i t}\left(r_{t} d t+\pi_{i t} \sigma_{t}\left(\theta_{t} d t+d B_{t}\right)\right)-c_{i t} d t .
\end{aligned}
$$

In the next, we denote by $\pi_{i t}^{\text {myopic }}$ the myopic (instantaneously mean variance efficient) portfolio given by

$$
\pi_{i t}^{\text {myopic }}=\frac{\delta_{i}+\theta_{t}}{\gamma_{i} \sigma_{t}}
$$

and we denote by $\pi_{i t}^{\text {hedging }}=\pi_{i t}-\pi_{i t}^{\text {myopic }}$ the hedging component of the optimal portfolio, i.e. the component that permits to hedge against future fluctuations of the market risk premium.

The following proposition characterizes the optimal portfolio and provides its asymptotic composition. 
Proposition 8.3 1. The optimal portfolio is given by

$$
\sigma_{t} \pi_{i t}=\theta_{t}+\frac{E_{t}\left[\int_{t}^{\infty}\left(b_{i} \delta_{i}+\left(b_{i}-1\right) \theta_{\tau}\right) M_{\tau} c_{i \tau} d \tau\right]}{E_{t}\left[\int_{t}^{\infty} M_{\tau} c_{i \tau} d \tau\right]}
$$

In particular,

$$
\min _{j} \theta_{j}+\min _{j}\left(b_{i} \delta_{i}+\left(b_{i}-1\right) \theta_{j}\right) \leq \sigma_{t} \pi_{i t} \leq \max _{j} \theta_{j}+\max _{j}\left(b_{i} \delta_{i}+\left(b_{i}-1\right) \theta_{j}\right)
$$

2. If we further assume that $\gamma_{i}>1$, for all $i$, then

$$
\lim _{t \rightarrow \infty} \pi_{i t}=\frac{\delta_{i}+\theta_{I_{K}}}{\sigma \gamma_{i}}
$$

3. Suppose risk aversion is homogeneous. The sign of $(1-b) \pi_{i t}^{\text {hedging }}$

- is positive if the sequences

$$
\begin{gathered}
b \sigma \delta_{i}+\frac{1}{2} b(1-b) \delta_{i}^{2}+b \rho_{i}-2 b^{2}\left(\max _{j} \theta_{j}+\delta_{i}\right) \delta_{i}, \\
b \sigma \delta_{i}+\frac{1}{2} b(1-b) \delta_{i}^{2}+b \rho_{i}-2 b^{2}\left(\min _{j} \theta_{j}+\delta_{i}\right) \delta_{i}
\end{gathered}
$$

are both anti-co-monotone with $\left(\delta_{i}\right)$;

- is negative if the sequences (13)-(14) are both co-monotone with $\left(\delta_{i}\right)$.

The asymptotic risky portfolio corresponds then, for each agent, to his optimal risky portfolio when facing an asset whose risk premium corresponds to the asymptotic risk premium of our heterogeneous economy, that is to say the risk premium that would prevail in the economy populated by agent $I_{K}$ only.

\section{Appendix}

\section{Proof of Proposition 3.1}

Immediate. 


\section{Proof of Lemma 4.1}

Let

$$
b_{i}=1 / \gamma_{i}
$$

denote the relative risk tolerance. The optimal consumption of investor $i$ is given by

$$
c_{i t}=e^{-\rho_{i} b_{i} t} M_{t}^{-b_{i}} Z_{i t}^{b_{i}} c_{i 0}=\left(c_{i 0}^{\gamma_{i}} M_{i t}\right)^{b_{i}} D_{t} M_{t}^{-b_{i}} .
$$

In equilibrium we require that

$$
\sum_{i=1}^{n} c_{i t}=D_{t}
$$

or equivalently

$$
\sum_{i}\left(c_{i 0}^{\gamma_{i}} M_{i t}\right)^{b_{i}} M_{t}^{-b_{i}}=1
$$

Let $F\left(a_{1}, \cdots, a_{n}\right)$ be the function defined as the unique solution to

$$
\sum_{i} F^{-b_{i}} a_{i}^{b_{i}}=1
$$

Then, a direct consequence of the equilibrium equation is that

$$
M=F\left(c_{10}^{\gamma_{1}} M_{1}, \cdots, c_{N 0}^{\gamma_{1}} M_{N}\right)
$$

Let $\Gamma \geq 1$ be such that $\Gamma b_{i}>1$ for all $i$ and $\gamma \leq 1$ be such that $\gamma b_{i} \leq 1$ for all $i$. We have $\sum_{i}\left(c_{i 0}^{\gamma_{i}} M_{i t}\right)^{b_{i}} M_{t}^{-b_{i}}=1$ which gives $\left(c_{i 0}^{\gamma_{i}} M_{i t}\right) M_{t}^{-1} \leq 1$ and

$$
\sum_{i}\left(c_{i 0}^{\gamma_{i}} M_{i t}\right)^{\frac{1}{\Gamma}} M_{t}^{-\frac{1}{\Gamma}} \geq 1 \geq \sum_{i}\left(c_{i 0}^{\gamma_{i}} M_{i t}\right)^{\frac{1}{\gamma}} M_{t}^{-\frac{1}{\gamma}}
$$

The bounds on $M_{t}$ follow from there.

\section{Proof of Corollary 4.1}

The first part is immediate. Note that $F\left(a_{1}, \cdots, a_{N}\right)>F\left(a_{1}^{\prime}, \cdots, a_{N}^{\prime}\right)$ whenever $a_{i}>a_{i}^{\prime}$ for $i=1, \cdots N$. The state price density $M_{t}$ is then decreasing in $W_{t}$ whenever all the state price densities $M_{i t}$ are decreasing in $W_{t}$ for $i=1, \cdots N$. The asymptotic results are immediate.

\section{Proof of Corollary 5.1}


By Lemma 4.1, $M_{t} \geq c_{I_{K} 0}^{\gamma_{I_{K}}} e^{-\rho_{I_{K}}{ }^{t}} Z_{I_{K} t} D_{t}^{-\gamma_{I_{K}}}$. Therefore, for $i \neq I_{K}$

$$
\begin{gathered}
c_{i t} D_{t}^{-1}=e^{-\rho_{i} b_{i} t} M_{t}^{-b_{i}} Z_{i t}^{b_{i}} c_{i 0} \leq e^{-\rho_{i} b_{i} t}\left(c_{I_{K} 0}^{\gamma_{I_{K}}} e^{-\rho_{I_{K}} t} Z_{I_{K} t} D_{t}^{-\gamma_{I_{K}}}\right)^{-b_{i}} Z_{i t}^{b_{i}} c_{i 0} D_{t}^{-1} \\
=\left(\frac{e^{-\rho_{i} t} c_{i 0}^{\gamma_{i}} Z_{i t}}{e^{-\rho_{I_{K}} t} c_{I_{K} 0}^{\gamma_{I_{K}}} Z_{I_{K} t}} D_{t}^{\gamma_{I_{K}}-\gamma_{i}}\right)^{b_{i}}=e^{b_{i}\left(\kappa_{I_{K}}-\kappa_{i}\right) t+b_{i}\left(\gamma_{j}-\gamma_{i}\right) \sigma W_{t}}
\end{gathered}
$$

By definition, $\kappa_{I_{K}}-\kappa_{i}<0$ and therefore $c_{i t} D_{t}^{-1}$ converges to zero by the law of large numbers. Since $\sum_{i} c_{i}=D$, we have $\lim _{t \rightarrow \infty} c_{I_{K} t} D_{t}^{-1}=1$. The limits when $W_{t}$ goes to $+\infty$ or $-\infty$ result directly from Corollary 4.1. Finally, we have $\omega_{i t}=c_{i 0}^{\gamma_{i}} M_{i} F^{-1} F_{a_{i}}$ and then

$$
\begin{aligned}
\frac{d \omega_{i t}\left(W_{t}\right)}{d W_{t}}=c_{i 0}^{\gamma_{i}} \frac{d M_{i t}}{d W_{t}} F^{-1} F_{a_{i}}-c_{i 0}^{\gamma_{i}} M_{i} F^{-2} & F_{a_{i}} \sum_{j} F_{a_{j}} c_{j 0}^{\gamma j} \frac{d M_{j t}}{d W_{t}} \\
& +c_{i 0}^{\gamma_{i}} M_{i} F^{-1} \sum_{j} F_{a_{i} a_{j}} c_{j 0}^{\gamma j} \frac{d M_{j t}}{d W_{t}} .
\end{aligned}
$$

If we differentiate the formula

$$
\sum_{k} F^{1-b_{k}} a_{k}^{b_{k}}-F=0
$$

with respect to $a_{i}$ and then with respect to $a_{j}$, we get

$$
\begin{aligned}
F_{a_{i} a_{j}}=\left(\left(1-b_{j}\right)+\left(1-b_{i}\right)\right) \omega_{i t} \omega_{j t} M_{t}\left(c_{i 0}^{\gamma_{i}} M_{i t} c_{j 0}^{\gamma_{j}} M_{j t}\right)^{-1} & \\
& \quad-M_{t}^{-1} \sum_{k}\left(1-b_{k}\right) \omega_{k t} F_{a_{i}} F_{a_{j}}+\delta_{i j}\left(b_{i}-1\right)\left(c_{i 0}^{\gamma_{i}} M_{i t}\right)^{-2} M_{t} \omega_{i t}
\end{aligned}
$$

where $\delta_{i j}$ is equal to 1 for $i=j$ and to 0 for $i \neq j$. Replacing in (17) and recombining the different terms leads to

$$
\frac{d \omega_{i t}\left(W_{t}\right)}{d W_{t}}=\omega_{i t}\left[b_{i}\left(\theta_{t}-\theta_{i}\right)-\sum_{j} \omega_{j t} b_{j}\left(\theta_{t}-\theta_{j}\right)\right] .
$$

\section{Proof of Proposition 6.1}

Assume that $M$ can be written in the representation

$$
d M_{t}=M_{t}\left(-r_{t} d t-\theta_{t} d W_{t}\right)
$$


The risk free rate is then given by $r_{t}$ and the market price of risk is given by $\theta_{t}$. Since

$$
M_{t}=F\left(c_{10}^{\gamma_{1}} M_{1 t}, \cdots, c_{N 0}^{\gamma_{N}} M_{N t}\right)
$$

we have,

$$
d M_{t}=\sum_{i} c_{i 0}^{\gamma_{i}} F_{a_{i}} d M_{i t}+\frac{1}{2} \sum_{i, j} c_{i 0}^{\gamma_{i}} c_{j 0}^{\gamma_{j}} F_{a_{i} a_{j}} d\left\langle M_{i t}, M_{j t}\right\rangle
$$

By definition,

$$
d M_{i t}=M_{i t}\left(-r_{i} d t-\theta_{i} d W_{t}\right)
$$

and the formula

$$
\theta_{t}=\sum_{i=1}^{N} \omega_{i t} \theta_{i}
$$

follows directly. As far as the risk free rate is concerned, we have

$$
d\left\langle M_{i t}, M_{j t}\right\rangle=M_{i t} M_{j t} \theta_{i} \theta_{j} d t
$$

which with (18) leads to

$$
\begin{aligned}
r_{t} & =\sum_{i=1}^{N} \omega_{i t} r_{i}-\left(\sum_{i=1}^{N} \theta_{i} \omega_{i t}\right)\left(\sum_{j=1}^{N} \theta_{j}\left(1-b_{j}\right) \omega_{j t}\right) \\
& +\frac{1}{2}\left(\sum_{k=1}^{N}\left(1-b_{k}\right) \omega_{k t}\right)\left(\sum_{i=1}^{N} \theta_{i} \omega_{i t}\right)^{2}+\frac{1}{2} \sum_{i=1}^{N}\left(1-b_{i}\right) \theta_{i}^{2} \omega_{i t} .
\end{aligned}
$$

\section{Proof of Corollary 6.1}

The first point is immediate.

Using the inequality

$$
a^{2}+b^{2} \geq 2 a b
$$

we get

$$
\begin{aligned}
\frac{1}{2}\left(\sum_{k} \mid\right. & \left.1-b_{k} \mid \omega_{k t}\right)\left(\sum_{i} \theta_{i} \omega_{i t}\right)^{2}+\frac{1}{2} \sum_{i}\left|1-b_{i}\right| \theta_{i}^{2} \omega_{i t} \\
& \geq\left(\sum_{k}\left|1-b_{k}\right| \omega_{k t}\right)^{1 / 2}\left(\sum_{i} \theta_{i} \omega_{i t}\right)\left(\sum_{i}\left|1-b_{i}\right| \theta_{i}^{2} \omega_{i t}\right)^{1 / 2} .
\end{aligned}
$$


Now, applying the Cauchy-Schwarz inequality

$$
\left(\sum_{i} x_{i}^{2}\right)^{1 / 2}\left(\sum_{i} y_{i}^{2}\right)^{1 / 2} \geq \sum_{i} x_{i} y_{i}
$$

to

$$
x_{i}=\left|1-b_{i}\right|^{1 / 2} \theta_{i} \omega_{i t}^{1 / 2} \text { and } y_{i}=\left|1-b_{i}\right|^{1 / 2} \omega_{i t}^{1 / 2}
$$

we get the first or the second inequality depending on the sign of the $\left(1-b_{i}\right)$ s. The limits are obtained from the behavior of the $\omega_{i} \mathrm{~s}$ in extreme states of the world.

The asymptotic results are immediate from Corollary 5.1.

\section{Proof of Corollary 6.2}

We have,

$$
\omega_{i t}=c_{i 0}^{\gamma_{i}} M_{i t} F^{-1} F_{a_{i}}
$$

and therefore

$$
\begin{aligned}
& \frac{d \omega_{i t}\left(W_{t}\right)}{d W_{t}} \\
= & c_{i 0}^{\gamma_{i}}\left(\frac{d M_{i t}}{d W_{t}} F^{-1} F_{a_{i}}-M_{i t} F^{-2} F_{a_{i}} \sum_{j} c_{j 0}^{\gamma_{j}} F_{a_{j}} \frac{d M_{j t}}{d W_{t}}+M_{i t} F^{-1} \sum_{j} c_{j 0}^{\gamma_{j}} F_{a_{i} a_{j}} \frac{d M_{j t}}{d W_{t}}\right) \\
= & -\omega_{i t} b_{i} \theta_{i}+\omega_{i t} \theta_{t}-\omega_{i t} \sum_{j} \omega_{j t}\left(1-b_{j}\right) \theta_{j}-\omega_{i t}\left(1-b_{i}\right) \theta_{t}+\omega_{i t} \theta_{t} \sum_{k}\left(1-b_{k}\right) \omega_{k t} .
\end{aligned}
$$

After some manipulations, we get

$$
\begin{aligned}
\frac{d \theta_{t}}{d W_{t}} & =\sum_{i} \theta_{i} \frac{d \omega_{i t}}{d W_{t}} \\
& =-\sum_{i} \omega_{i t} b_{i}^{2} \theta_{i}^{2}+\theta_{t}^{2}-2 \theta_{t} \sum_{i} \omega_{i t}\left(1-b_{i}\right) \theta_{i}+\theta_{t}^{2} \sum_{k}\left(1-b_{k}\right) \omega_{k t} \\
& =2\left(\sum_{j} \omega_{j t} \theta_{j}\right)\left(\sum_{j} \omega_{j t} b_{j} \theta_{j}\right)-\left(\sum_{j} \omega_{j t} \theta_{j}^{2}\right)\left(\sum_{j} \omega_{j t} b_{j}\right)-\sum_{j} \omega_{j t} b_{j} \theta_{j}^{2} .
\end{aligned}
$$

Now, applying Cauchy-Schwarz, we get

$$
\left(\sum_{j} \omega_{j t} b_{j} \theta_{j}^{2}\right)\left(\sum_{j} \omega_{j t} b_{j}\right) \geq\left(\sum_{j} \omega_{j t} b_{j} \theta_{j}\right)^{2}
$$


and

$$
\sum_{j} \omega_{j t} \theta_{j}^{2} \geq\left(\sum_{j} \omega_{j t} \theta_{j}\right)^{2}
$$

and therefore

$$
\begin{aligned}
& \left(\sum_{j} \omega_{j t} \theta_{j}^{2}\right)\left(\sum_{j} \omega_{j t} b_{j}\right)+\sum_{j} \omega_{j t} b_{j} \theta_{j}^{2} \\
& \geq 2\left(\left(\sum_{j} \omega_{j t} \theta_{j}^{2}\right)\left(\sum_{j} \omega_{j t} b_{j}\right)\left(\sum_{j} \omega_{j t} b_{j} \theta_{j}^{2}\right)\right)^{1 / 2} \\
& \geq 2\left(\sum_{j} \omega_{j t} \theta_{j}\right)\left(\sum_{j} \omega_{j t} b_{j} \theta_{j}\right)
\end{aligned}
$$

which is what had to be proved.

\section{Proof of Corollary 6.3}

$$
\begin{aligned}
\frac{d r_{t}}{d W_{t}}=\frac{d}{d W_{t}}\left[\sum_{i} \omega_{i} r_{i}-\theta_{t}\left(\sum_{j} \theta_{j}\left(1-b_{j}\right) \omega_{j t}\right)\right. & +\frac{1}{2}\left(\sum_{k}\left(1-b_{k}\right) \omega_{k t}\right) \theta_{t}^{2} \\
& \left.+\frac{1}{2} \sum_{i}\left(1-b_{i}\right) \theta_{i}^{2} \omega_{i t}\right]
\end{aligned}
$$

Given $X=\left(x_{1}, \ldots, x_{N}\right)$, denote

$$
\mathcal{E}(x)=\sum_{i} \omega_{i t} x_{i}
$$

Since the weights sum up to one, this is an expectation on $\{1, \cdots, n\}$ and we can also define $\operatorname{Cov}_{\omega}$ and $\operatorname{Var}_{\omega}$. Let

$$
R=\left(r_{i}\right)_{i=1, \cdots, N}, \quad \beta=\left(b_{i}\right)_{i=1, \cdots, N} \text { and } \Theta=\left(\theta_{i}\right)_{i=1, \cdots, N} .
$$

Then, a direct but tedious calculation implies that 


$$
\begin{aligned}
\frac{d r_{t}}{d W_{t}}=-\operatorname{Cov}_{\omega}(\beta \Theta, R)+\mathcal{E}(\Theta) \operatorname{Cov}_{\omega}(\beta, R) \\
+\mathcal{E}(\Theta) \operatorname{Cov}_{\omega}(\beta \Theta, \Theta)-\mathcal{E}(\Theta) \operatorname{Var}(\beta \Theta)-\mathcal{E}(\Theta)^{2} \operatorname{Cov}_{\omega}(\Theta, \beta)+\mathcal{E}(\Theta)^{2} \operatorname{Cov}_{\omega}(\Theta \beta, \beta) \\
\quad+\frac{1}{2} \mathcal{E}(\Theta)^{2}\left(\operatorname{Cov}_{\omega}(\Theta \beta, \beta)-\mathcal{E}(\Theta) \operatorname{Var}_{\omega}(\beta)\right) \\
+\frac{1}{2}\left(\operatorname{Cov}_{\omega}\left(\beta(\beta-1) \Theta^{2}, \Theta\right)+\operatorname{Cov}_{\omega}(\Theta, \beta) \mathcal{E}\left((1-\beta) \Theta^{2}\right)\right. \\
+\operatorname{Cov}_{\omega}(\beta, \Theta)\left(2 \mathcal{E}(\Theta) \mathcal{E}(\beta \Theta)-\mathcal{E}\left(\Theta^{2}\right) \mathcal{E}(\beta)-\mathcal{E}\left(\beta \Theta^{2}\right)\right)
\end{aligned}
$$

In particular, if risk aversion is homogeneous (that is, $\beta=(b, \cdots, b)$ ) then we get

$$
\begin{aligned}
\frac{d r_{t}}{d W_{t}}=-b \operatorname{Cov}_{\omega}(\Theta, R) & +b(b-1) \frac{1}{2} \operatorname{Cov}_{\omega}\left(\Theta^{2}, \Theta\right) \\
= & -\operatorname{Cov}_{\omega}\left(-b \sigma\left(\delta_{i}\right)+0.5 b(b-1)\left(\delta_{i}^{2}\right)-b \rho_{i},\left(\delta_{i}\right)\right)
\end{aligned}
$$

and the claim follows.

\section{Proof of Proposition 7.1}

We have $E_{t}\left[M_{i T}\right]=\exp \left(-r_{i}(T-t)-\kappa_{i} t-\theta_{i} W_{t}\right)$. From (4) we obtain $\lim _{T \rightarrow \infty} \frac{1}{T} \ln E_{t}\left[M_{T}\right]=r_{I_{0}}$ and since $B(t, T)=\frac{1}{M_{t}} E_{t}\left[M_{T}\right]$, we get

$$
\lim _{T \rightarrow \infty} Y(t, T)=r_{I_{0}}
$$

\section{Proof of Proposition 7.2}

For $\lambda \in\left(\lambda_{j-1}, \lambda_{j}\right)$ and $t=\lambda T$ we have

$$
\lim _{T \rightarrow \infty} \frac{E_{t}\left[M_{i T}\right]}{E_{t}\left[M_{I_{j} T}\right]} \rightarrow 0
$$

for $i \neq I_{j}$. The asymptotic behavior of $E_{t}\left[M_{\alpha t}\right]$ for $\alpha \in\left(\frac{1}{\lambda_{j+1}}, \frac{1}{\lambda_{j}}\right)$ derives directly from there and from Equation (4). By definition, $B(t, T)$ is equal to $\frac{1}{M_{t}} E_{t}\left[M_{T}\right]$ which gives the asymptotic behavior of $B(t, \alpha t)$.

Since $\frac{B_{t}}{t}$ converges to 0 almost surely we have, for $\alpha \in\left(\frac{1}{\lambda_{j}}, \frac{1}{\lambda_{j-1}}\right)$,

$$
\lim _{t \rightarrow \infty} Y(t, \alpha t)=\frac{1}{\alpha-1}\left[\kappa_{I_{K}}-\alpha l_{I_{j}}(1 / \alpha)\right]
$$


and the asymptotic instantaneous forward rate at date $\alpha t$ seen from date $t$ is given by $r_{I_{j}}$.

As far as the uniform convergence is concerned, note that

$$
\frac{d}{d \alpha} Y(t, \alpha t)=\frac{t}{(\alpha t-t)^{2}} \ln B(t, \alpha t)-\frac{1}{\alpha t-t} \frac{\frac{d}{d \alpha} B(t, \alpha t)}{B(t, \alpha t)} .
$$

Let $\tilde{P}$ be the equivalent martingale measure, corresponding to $M_{t}$. We have $B(t, \alpha t)=E_{t}^{\tilde{P}}\left[\exp -\int_{t}^{\alpha t} r_{s} d s\right]$ and $\frac{d}{d \alpha} B(t, \alpha t)=E_{t}^{\tilde{P}}\left[-t r_{\alpha t} \exp -\int_{t}^{\alpha t} r_{s} d s\right]$. If we denote by $Q_{t}$ the probability defined by its density $\frac{d Q_{t}}{d \tilde{P}}=\frac{\exp -\int_{t}^{\alpha t} r_{s} d s}{E\left[\exp -\int_{t}^{\alpha t} r_{s} d s\right]}$ then we have $\frac{d}{d \alpha} Y(t, \alpha t)=\frac{1}{(\alpha-1)}\left(-Y(t, \alpha)+E_{t}^{Q t}\left[r_{\alpha t}\right]\right)$.

We also have

$$
\begin{aligned}
& \left(\sum_{i} c_{i 0}^{\gamma_{i} / \gamma}\right)^{\frac{1}{\gamma}} \exp \left[(\alpha t-t) \min _{i} l_{i}(0)\right] \\
& \leq B(t, \alpha t) \leq\left(\sum_{i} c_{i 0}^{\gamma_{i} / \Gamma}\right)^{\frac{1}{\Gamma}} \exp \left[(\alpha t-t) \max _{i} l_{i}(0)\right] \\
& \frac{1}{t-\alpha t} \frac{1}{\gamma} \ln \left(\sum_{i} c_{i 0}^{\gamma_{i} / \gamma}\right)-\max _{i} l_{i}(0) \\
& \leq Y(t, \alpha t) \leq \frac{1}{t-\alpha t} \frac{1}{\Gamma} \ln \left(\sum_{i} c_{i 0}^{\gamma_{i} / \Gamma}\right)-\min _{i} l_{i}(0)
\end{aligned}
$$

which gives us that $\frac{1}{\alpha-1} Y(t, \alpha t)$ is bounded on the compact subsets of $(1, \infty)$. Using the expression for $r_{t}$ given by Proposition 6.1 we also have that $r_{\alpha t}$ and hence $\frac{1}{\alpha-1} E_{t}^{Q_{t}}\left[r_{\alpha t}\right]$ are bounded on the compact subsets of $(1, \infty)$. The mappings $\alpha \rightarrow Y(t, \alpha t)$ are then uniformy Lipschitz on the compact subsets of $(1, \infty)$ and the convergence of $Y(t, \alpha t)$ to $Y(\alpha)$ is then uniform on the compact subsets of $(1, \infty)$.

\section{Proof of Proposition 8.1}

1. We can rewrite the defining identity for the stock price as

$$
\int_{0}^{t} M_{\tau} D_{\tau} d \tau+S_{t} M_{t}=E_{t}\left[\int_{0}^{\infty} M_{\tau} D_{\tau} d \tau\right] .
$$

Thus,

$$
M_{t} D_{t} d t+d\left(S_{t} M_{t}\right)=\gamma_{t} d B_{t}
$$


for an adapted process $\gamma_{t}$ given by

$$
\gamma_{t}=E_{t}\left[\int_{t}^{\infty} \mathcal{D}_{t}\left(M_{\tau} D_{\tau}\right) d \tau\right]
$$

where $\mathcal{D}$ denotes Malliavin derivatives. Using Ito's formula, we get

$$
\gamma_{t}=S_{t} M_{t}\left(-\theta_{t}+\sigma_{t}^{S}\right)
$$

Let us now calculate $\gamma_{t}$. We have

$$
\mathcal{D}_{t}\left(M_{\tau} D_{\tau}\right)=\mathcal{D}_{t}\left(M_{\tau}\right) D_{\tau}+M_{\tau} \sigma D_{\tau}
$$

and

$$
\mathcal{D}_{t}\left(M_{\tau}\right)=\mathcal{D}_{t}\left(F\left(c_{10}^{\gamma_{1}} M_{1 \tau}, \cdots, c_{N 0}^{\gamma_{N}} M_{N \tau}\right)\right)=\sum_{i} c_{i 0}^{\gamma_{1}} F_{a_{i}} \mathcal{D}_{t}\left(M_{i \tau}\right)=-M_{\tau} \theta_{\tau}
$$

Thus,

$$
\gamma_{t}=E_{t}\left[\int_{t}^{\infty}\left(\sigma-\theta_{\tau}\right) M_{\tau} D_{\tau} d \tau\right]
$$

which gives $\sigma_{t}^{S}$. The derivation of the upper and lower bounds is straightforward.

2. Since

$$
\theta_{t}=\sum_{i} \omega_{i t} \theta_{i}
$$

we get

$$
\frac{E_{t}\left[\theta_{t+u} M_{t+u} D_{t+u}\right]}{E_{t}\left[M_{t+u} D_{t+u}\right]}-\theta_{I_{K}}=\sum_{i \neq I_{K}} \theta_{i} \frac{E_{t}\left[\omega_{i t+u} M_{t+u} D_{t+u}\right]}{E_{t}\left[M_{t+u} D_{t+u}\right]} .
$$

By (16), we get

$$
\omega_{i, t+u} \leq \frac{b_{i}}{\min _{i} b_{i}} c_{i t+u} D_{t+u}^{-1} \leq e^{-\psi_{i}(t+u)+\eta_{i} W_{t+u}}
$$

for some $\psi_{i}>0$. Now, the same argument as in the proof of Proposition 7.2 (based on Lemma 4.1) implies that

$$
\lim _{t \rightarrow \infty} \frac{E_{t}\left[e^{-\psi_{i}(t+u)+\eta_{i} W_{t+u}} M_{t+u} D_{t+u}\right]}{E_{t}\left[e^{-\psi_{i}(t+u)+\eta_{i} W_{t+u}} M_{t+u}^{I_{K}} D_{t+u}\right]}=1
$$


and from the law of large numbers we have

$$
\lim _{t \rightarrow \infty} \frac{E_{t}\left[e^{-\psi_{i}(t+u)+\eta_{i} W_{t+u}} M_{t+u} D_{t+u}\right]}{E_{t}\left[M_{t+u} D_{t+u}\right]}=0
$$

for all $i \neq I_{K}$. With (27), this leads to

$$
\frac{E_{t}\left[\theta_{t+u} M_{t+u} D_{t+u}\right]}{E_{t}\left[M_{t+u} D_{t+u}\right]} \rightarrow \theta_{I_{K}} .
$$

and then to

$$
\lim _{t \rightarrow \infty} \sigma_{t}^{S}=\sigma
$$

3. We have

$$
\frac{S_{t}}{D_{t}}=E_{t}\left[\int_{t}^{\infty} \frac{M_{\tau} D_{\tau}}{M_{t} D_{t}} d \tau\right] .
$$

Note we can rewrite the aggregate consumption condition as

$$
1=\sum_{i=1}^{N}\left(\frac{M_{\tau}}{M_{t}}\right)^{-b_{i}}\left(\frac{M_{i \tau}}{M_{i t}}\right)^{b_{i}}\left(c_{i t} D_{t}^{-1}\right)
$$

Then, the same argument as in the proof of Lemma 4.1 gives us

$$
\left(\sum_{i}\left(\left(c_{i t} D_{t}^{-1}\right)^{\gamma_{i}} \frac{M_{i \tau}}{M_{i t}}\right)^{1 / \gamma}\right)^{\gamma} \leq \frac{M_{\tau}}{M_{t}} \leq\left(\sum_{i}\left(\left(c_{i t} D_{t}^{-1}\right)^{\gamma_{i}} \frac{M_{i \tau}}{M_{i t}}\right)^{1 / \Gamma}\right)^{\Gamma}
$$

for $\Gamma \geq 1$ such that $\Gamma b_{i}>1$ for all $i$ and for $\gamma \leq 1$ such that $\gamma b_{i} \leq 1$ for all $i$. Similarly, we have

$$
\begin{aligned}
\left(\sum_{i}\left(\left(c_{i t} D_{t}^{-1}\right)^{\gamma_{i}} E_{t}\left[\frac{D_{\tau} M_{i \tau}}{D_{t} M_{i t}}\right]\right)^{1 / \gamma}\right)^{\gamma} \leq E_{t}\left[\frac{D_{\tau} M_{\tau}}{D_{t} M_{t}}\right] \\
\leq\left(\sum_{i}\left(\left(c_{i t} D_{t}^{-1}\right)^{\gamma_{i}} E_{t}\left[\frac{D_{\tau} M_{i \tau}}{D_{t} M_{i t}}\right]\right)^{1 / \Gamma}\right)^{\Gamma}
\end{aligned}
$$

Since all finite dimensional norms are equivalent, there exist constants $K_{1}>$ $K_{2}>0$ such that

$K_{2} \sum_{i}\left(c_{i t} D_{t}^{-1}\right)^{\gamma_{i}} E_{t}\left[\frac{D_{\tau} M_{i \tau}}{D_{t} M_{i t}}\right] \leq E_{t}\left[\frac{D_{\tau} M_{\tau}}{D_{t} M_{t}}\right] \leq K_{1} \sum_{i}\left(c_{i t} D_{t}^{-1}\right)^{\gamma_{i}} E_{t}\left[\frac{D_{\tau} M_{i \tau}}{D_{t} M_{i t}}\right]$. 
Pick now an $\epsilon>0$ and let $T>0$ be so large that

$$
\sum_{i}\left(\frac{S}{D}\right)_{i} e^{-T\left(\frac{S}{D}\right)_{i}^{-1}}<\epsilon
$$

then by (32) we have

$$
E_{t}\left[\int_{t+T}^{\infty} \frac{M_{\tau} D_{\tau}}{M_{t} D_{t}} d \tau\right] \leq K_{1} \epsilon
$$

for all $t>0$. Now, the same argument as in the proof of Proposition 7.2 implies that, for any $\theta>0$,

$$
E_{t}\left[\frac{D_{t+\theta} M_{t+\theta}}{D_{t} M_{t}}\right] \rightarrow e^{-\theta\left(\frac{S}{D}\right)_{I_{K}}^{-1}}
$$

Consequently, because of uniform boundedness, guaranteed by (32), we can interchange limit and integration and we have

$$
E_{t}\left[\int_{0}^{T} \frac{D_{t+\theta} M_{t+\theta}}{D_{t} M_{t}} d \theta\right] \rightarrow \int_{0}^{T} e^{-\theta\left(\frac{S}{D}\right)_{I_{K}}^{-1}} d \theta .
$$

Since $\epsilon>0$ is arbitrary and $T$ can be made arbitrarily large, we are done.

4. After some algebraic manipulations, we have

$$
\frac{S_{t}}{D_{t}}=E_{t}\left[\int_{t}^{+\infty} e^{-\int_{0}^{t} \zeta_{s} d s} \frac{N_{t+\tau}}{N_{t}} d \tau\right]
$$

where

$$
\zeta_{t}=r_{t}+\theta_{t} \sigma-\mu
$$

is the risk-adjusted discount rate and

$$
N_{t}=e^{-0.5 \int_{0}^{t}\left(\theta_{s}-\sigma\right)^{2} d s-\int_{0}^{t}\left(\theta_{s}-\sigma\right) d W_{s}}
$$

is an exponential martingale. Thus, $N_{t}$ is a density process of a measure $d \nu$ and, under this measure, we can rewrite

$$
\frac{S_{t}}{D_{t}}=E_{t}^{\nu}\left[\int_{t}^{+\infty} e^{-\int_{t}^{\tau} \zeta_{s} d s} d \tau\right] .
$$

Lemma 1 in Mele (2007) implies that $\frac{S_{t}}{D_{t}}$ is procyclical (countercyclical) if and only if $\zeta_{t}$ is countercyclical (procyclical). 
Furthermore, if $g$ is such that

$$
\frac{S_{t}}{D_{t}}=g\left(t, W_{t}\right)
$$

then, by the Ito formula,

$$
\sigma_{t}^{S}=\sigma+\frac{\partial g / \partial W_{t}}{g}
$$

and hence excess volatility is positive if and only if $g$ is monotone increasing. Thus, we need to check cyclicality of the risk-adjusted discount rate $\zeta_{t}$.

We have

$$
\frac{\partial \zeta_{t}}{\partial W_{t}}=\frac{\partial r_{t}}{\partial W_{t}}+\frac{\partial \theta_{t}}{\partial W_{t}} \sigma
$$

We have

$$
\frac{\partial \theta_{t}}{\partial W_{t}}=-2 b \operatorname{Var}^{\omega_{i}}\left(\delta_{i}\right)
$$

and hence we need that

$$
\begin{aligned}
\operatorname{Cov}^{\omega_{i}}\left(b \sigma \delta_{i}+0.5 b(1-b) \delta_{i}^{2}+\right. & \left.b \rho_{i}, \delta_{i}\right)-2 b \sigma \operatorname{Var}^{\omega_{i}}\left(\delta_{i}\right) \\
& =\operatorname{Cov}^{\omega_{i}}\left(0.5 b(1-b) \delta_{i}^{2}+b \rho_{i}-b \sigma \delta_{i}, \delta_{i}\right) .
\end{aligned}
$$

\section{Proof of Proposition $\mathbf{8 . 2}$}

Introduce a new measure $d P^{(\alpha)}$ such that its restriction on $\mathcal{F}_{T}$ is given by

$$
d P_{T}^{(\alpha)}=\frac{D_{T}^{\alpha}}{E\left[D_{T}^{\alpha}\right]} d P_{T}
$$

where $P_{T}$ is the physical measure. Under the measure $d P^{(\alpha)}, W_{t}$ has drift $\sigma \alpha, d W_{t}=d W_{t}^{(\alpha)}+\sigma^{2} \alpha d t$, and $D_{t}$ has a drift $\mu+\sigma^{2} \alpha$. By the Kolmogorov extension theorem, we can extend this measure to the set of infinite paths. Then, we can rewrite the expression for $R(t, T)$ as

$$
R(t, T)=\frac{E_{t}\left[D_{T}\right]}{D_{t}} \frac{D_{t}}{S_{t}} E_{t}^{(1)}\left[\frac{S_{T}}{D_{T}} e^{\int_{t}^{T} D_{s} S_{s}^{-1} d s}\right]
$$

By (32) we have,

$$
L_{2} \equiv K_{2} n^{-\max _{i} \gamma_{i}} \min _{i} S_{i} \leq \frac{S_{t}}{D_{t}} \leq K_{1} \sum_{i} S_{i} \equiv L_{1} .
$$


Consequently, $S_{t} / D_{t}$ is uniformly bounded both from zero and infinity and hence

$$
\begin{aligned}
& \mu+\left(\log \left(L_{2} / L_{1}\right)\right.\left.+\log E_{t}^{(1)}\left[e^{\int_{t}^{T} D_{s} S_{s}^{-1} d s}\right]\right) \\
& \leq \lim \inf _{T \rightarrow \infty}(T-t)^{-1} \log R(t, T) \\
& \leq \mu+(T-t)^{-1}\left(\log \left(L_{1} / L_{2}\right)+\log E_{t}^{(1)}\left[e^{\int_{t}^{T} D_{s} S_{s}^{-1} d s}\right]\right)
\end{aligned}
$$

and hence

$$
\begin{gathered}
\mu+\lim \inf _{T \rightarrow \infty}(T-t)^{-1} \log E_{t}^{(1)}\left[e^{\int_{t}^{T} D_{s} S_{s}^{-1} d s}\right] \\
\leq \lim \inf _{T \rightarrow \infty}(T-t)^{-1} \log R(t, T) \leq \lim \sup _{T \rightarrow \infty}(T-t)^{-1} \log R(t, T) \\
\leq \mu+\lim \sup _{T \rightarrow \infty}(T-t)^{-1} \log E_{t}^{(1)}\left[e^{\int_{t}^{T} D_{s} S_{s}^{-1} d s}\right] .
\end{gathered}
$$

The optimal consumption of agent $i$ can be rewritten as

$$
c_{i t}=e^{-\rho_{i}^{(\alpha)} b_{i} t} M_{t}^{-b_{i}}\left(Z_{i t}^{(\alpha)}\right)^{b_{i}} c_{i 0}
$$

where

$$
\rho_{i}^{(\alpha)}=\rho_{i}-\delta_{i} \sigma^{2} \alpha \quad \text { and } \quad Z_{i t}^{(\alpha)}=e^{\delta_{i} W_{t}^{(\alpha)}-\frac{1}{2} \delta_{i}^{2} t} .
$$

Thus, under this new measure everything looks the same, apart from the fact that agents have different discount rates. Note that the stock price is still calculated under the original, physical measure, but we can rewrite it as

$$
\begin{aligned}
\frac{S_{t}}{D_{t}}=\frac{1}{M_{t} D_{t}} E_{t}\left[\int_{t}^{\infty} M_{\tau} D_{\tau} d \tau\right] & =\frac{1}{M_{t} D_{t}} \int_{t}^{\infty} E_{t}\left[D_{\tau}^{\alpha}\right] \frac{E_{t}\left[M_{\tau} D_{\tau}^{1-\alpha} D_{t}^{\alpha}\right]}{E_{t}\left[D_{\tau}^{\alpha}\right]} d \tau \\
= & \frac{1}{M_{t} D_{t}} \int_{t}^{\infty} E_{t}\left[D_{\tau}^{\alpha}\right] E_{t}^{(\alpha)}\left[M_{\tau} D_{\tau}^{1-\alpha}\right] d \tau
\end{aligned}
$$

We define agent $A(\alpha)$ as (being the analog of agent $I_{K}$ )

$\left(\rho_{A}^{(\alpha)}+\gamma_{A}\left(\mu+\sigma^{2} \alpha-0.5 \sigma^{2}\right)+0.5 \delta_{A}^{2}\right)=\min _{i}\left(\rho_{i}^{(\alpha)}+\gamma_{i}\left(\mu+\sigma^{2} \alpha-0.5 \sigma^{2}\right)+0.5 \delta_{i}^{2}\right)$

By Corollary 4.1, under the measure $P^{(\alpha)}$, we have

$$
M_{t} \sim c_{I_{K} 0}^{\gamma_{A(\alpha)}} e^{-\rho_{A(\alpha)} t} Z_{A(\alpha) t} D_{t}^{-\gamma_{A(\alpha)}}
$$


Similarly, in complete analogy with the proof of Proposition 8.1,

$$
E_{t}^{(\alpha)}\left[M_{t+u} D_{t+u}^{1-\alpha}\right] \sim E_{t}^{(\alpha)}\left[M_{t+u}^{(\alpha)} D_{t+u}^{1-\alpha}\right]
$$

under $P^{(\alpha)}$ and we obtain that

$$
\lim _{t \rightarrow \infty} \frac{S_{t}}{D_{t}}=S_{A(\alpha)} \quad P^{(\alpha)}-a . s .
$$

by the same argument as in the proof of Proposition 8.1.

Now, Jensen's inequality implies

$$
\begin{array}{r}
\log E_{t}\left[S_{T} e^{\int_{t}^{T} D_{s} S_{s}^{-1} d s}\right]=\log E_{t}\left[D_{T}^{\alpha}\right]+\log \frac{E_{t}\left[D_{T}^{\alpha} D_{T}^{-\alpha} S_{T} e^{\int_{t}^{T} D_{s} S_{s}^{-1} d s}\right]}{E_{t}\left[D_{T}^{\alpha}\right]} \\
=\log E_{t}\left[D_{T}^{\alpha}\right]+\log E_{t}^{\alpha}\left[\left(D_{T}^{1-\alpha} \frac{S_{T}}{D_{T}} e^{\int_{t}^{T} D_{s} S_{s}^{-1} d s}\right)\right] \\
\geq E_{t}^{\alpha}\left[\log \left(S_{T} / D_{T}\right)\right]+\int_{t}^{T} E_{t}^{(\alpha)}\left[\left(D_{s} / S_{s}\right)\right] d s+E_{t}^{(\alpha)}\left[\log \left(D_{T}^{1-\alpha}\right)\right]+\log E_{t}\left[D_{T}^{\alpha}\right]
\end{array}
$$

We have

$$
\begin{aligned}
\log E_{t}\left[D_{T}^{\alpha}\right]= & E_{t}\left[e^{\alpha\left(\left(\mu-0.5 \sigma^{2}\right) T+\sigma W_{T}\right)}\right] \\
= & \log \left(e^{\sigma \alpha W_{t}} e^{0.5(1-\lambda) T \alpha^{2} \sigma^{2}+T \alpha\left(\mu-0.5 \sigma^{2}\right)}\right) \\
& =\sigma \alpha W_{t}+T \alpha\left(\mu+0.5 \sigma^{2}((1-\lambda) \alpha-1)\right)
\end{aligned}
$$

and

$$
\begin{aligned}
& E_{t}^{(\alpha)}\left[\log \left(D_{T}^{1-\alpha}\right)\right]=E_{t}^{\alpha}\left[\log \left(e^{(1-\alpha)\left(\left(\mu+(\alpha-0.5) \sigma^{2}\right) T+\sigma W_{T}^{(\alpha)}\right.}\right)\right] \\
& \quad=(1-\alpha)\left(\left(\mu+(\alpha-0.5) \sigma^{2}\right) T+(1-\alpha) \sigma\left(W_{t}-\sigma^{2} \alpha \lambda T\right)\right.
\end{aligned}
$$

Since $S_{T} / D_{T}$ converges to $S_{A(\alpha)} P^{(\alpha)}$-almost surely, a slight modification of the proof of Proposition 8.1 implies that in fact

$$
\lim _{T \rightarrow \infty}(1-\lambda)^{-1} T^{-1} \int_{\lambda T}^{T} E_{\lambda T}^{(\alpha)}\left[\left(D_{s} / S_{s}\right)\right] d s=S_{A(\alpha)}^{-1}
$$

and

$$
\lim _{\rightarrow \infty} T^{-1} E_{t}^{\alpha}\left[\log \left(S_{T} / D_{T}\right)\right]=0
$$


and, by the law of large numbers,

$$
\lim _{T \rightarrow \infty} T^{-1} \log S_{t}=\lim _{T \rightarrow \infty} \log D_{t}=\lambda\left(\mu-0.5 \sigma^{2}\right)
$$

Hence,

$$
\begin{aligned}
& \lim \inf _{T \rightarrow \infty} T^{-1} \log R(t, T) \geq-\lambda\left(\mu-0.5 \sigma^{2}\right) \\
+ & \alpha\left(\mu+0.5 \sigma^{2}((1-\lambda) \alpha-1)\right)+(1-\alpha)\left(\left(\mu+(\alpha-0.5) \sigma^{2}\right)+(1-\lambda) S_{A(\alpha)}^{-1}\right.
\end{aligned}
$$

\section{Proof of Proposition 8.3}

1. By the budget constraint, the wealth is given by the present value of future consumption, that is

$$
M_{t} w_{i t}=E_{t}\left[\int_{t}^{\infty} M_{\tau} c_{i \tau} d \tau\right]=c_{i 0} E_{t}\left[\int_{t}^{\infty} e^{-\rho_{i} b_{i} \tau} M_{\tau}^{1-b_{i}} e^{b_{i}\left(\delta_{i} W_{\tau}-\frac{1}{2} \delta_{i}^{2} \tau\right)} d \tau\right] .
$$

Similarly to what we did with the stock price (Proof of Proposition (8.1), we get that

$$
\int_{0}^{t} M_{\tau} c_{i \tau} d \tau+M_{t} w_{i t}=E_{t}\left[\int_{0}^{\infty} M_{\tau} c_{i \tau} d \tau\right]
$$

is a martingale and hence

$$
M_{t} c_{i t} d t+w_{i t} d M_{t}+M_{t} d w_{i t}+d\left\langle M_{t}, w_{i t}\right\rangle=\gamma_{t} d W_{t}
$$

where, by the Clark-Ocone formula,

$$
\gamma_{t}=E_{t}\left[\mathcal{D}_{t}\left(M_{\tau} c_{i \tau}\right) d \tau\right]
$$

By the Ito's formula,

$$
\gamma_{t}=w_{i t} M_{t}\left(-\theta_{t}+\sigma_{t} \pi_{i t}\right)
$$

Now, by the rules for Malliavin derivatives,

$$
\begin{aligned}
& \mathcal{D}_{t}\left(M_{\tau} c_{i \tau}\right)=\mathcal{D}_{t}\left(e^{-\rho_{i} b_{i} \tau} M_{\tau}^{1-b_{i}} e^{b_{i}\left(\delta_{i} W_{\tau}-\frac{1}{2} \delta_{i}^{2} \tau\right)}\right) \\
& \begin{aligned}
=\left(1-b_{i}\right) e^{-\rho_{i} b_{i} \tau} M_{\tau}^{-b_{i}} \mathcal{D}_{t}\left(M_{\tau}\right) e^{b_{i}\left(\delta_{i} W_{\tau}-\frac{1}{2} \delta_{i}^{2} \tau\right)} & +e^{-\rho_{i} b_{i} \tau} M_{\tau}^{1-b_{i}} b_{i} \delta_{i} e^{b_{i}\left(\delta_{i} W_{\tau}-\frac{1}{2} \delta_{i}^{2} \tau\right)} \\
=-\left(1-b_{i}\right) e^{-\rho_{i} b \tau} M_{\tau}^{-b_{i}} \theta_{\tau} M_{\tau} e^{b_{i}\left(\delta_{i} W_{\tau}-\frac{1}{2} \delta_{i}^{2} \tau\right)} & +e^{-\rho_{i} b_{i} \tau} M_{\tau}^{1-b_{i}} b_{i} \delta_{i} e^{b_{i}\left(\delta_{i} W_{\tau}-\frac{1}{2} \delta_{i}^{2} \tau\right)} \\
& =\left(b_{i} \delta_{i}+\left(b_{i}-1\right) \theta_{\tau}\right) M_{\tau} c_{i \tau}
\end{aligned}
\end{aligned}
$$


Thus,

$$
\gamma_{t}=E_{t}\left[\int_{t}^{\infty}\left(b_{i} \delta_{i}+\left(b_{i}-1\right) \theta_{\tau}\right) M_{\tau} c_{i \tau} d \tau\right]
$$

and we get

$$
\sigma_{t} \pi_{i t}=\theta_{t}+\frac{E_{t}\left[\int_{t}^{\infty}\left(b_{i} \delta_{i}+\left(b_{i}-1\right) \theta_{\tau}\right) M_{\tau} c_{i \tau} d \tau\right]}{E_{t}\left[\int_{t}^{\infty} M_{\tau} c_{i \tau} d \tau\right]} .
$$

2. Suppose now that $\gamma_{i}>1$. Let $\Gamma \geq 1$ be such that $\Gamma b_{j} /\left(1-b_{i}\right)>1$ for all $j$ and $\gamma \leq 1$ be such that $\gamma b_{j} /\left(1-b_{i}\right) \leq 1$ for all $j$. Then, the same argument as in the proof of Lemma 4.1 gives us

$$
\begin{gathered}
\left(\sum_{j}\left(c_{j 0}^{\gamma_{j}\left(1-b_{i}\right)} E_{t}\left[e^{-\rho_{j}\left(1-b_{i}\right) T} Z_{j T}^{1-b_{i}} Z_{i T}^{b_{i}} D_{T}^{-\gamma_{j}\left(1-b_{i}\right)}\right]\right)^{1 / \gamma}\right)^{\gamma} \leq E_{t}\left[M_{T}^{1-b_{i}} Z_{i T}^{b_{i}}\right] \\
\leq\left(\sum_{j}\left(c_{j 0}^{\gamma_{j}\left(1-b_{i}\right)} E_{t}\left[e^{-\rho_{j}\left(1-b_{i}\right) T} Z_{j T}^{1-b_{i}} Z_{i T}^{b_{i}} D_{T}^{-\gamma_{j}\left(1-b_{i}\right)}\right]\right)^{1 / \Gamma}\right)^{\Gamma}
\end{gathered}
$$

Denote

$$
c_{i t}^{\left(I_{K}\right)}=e^{-\rho_{i} b_{i} \tau}\left(M_{\tau}^{\left(I_{K}\right)}\right)^{-b_{i}} Z_{i t}^{b_{i}}
$$

Note that

$$
\begin{aligned}
& \frac{E_{t}\left[M_{t+u}^{\left(I_{K}\right)} c_{i t+u}^{I_{K}}\right]}{M_{t}^{\left(I_{K}\right)} c_{i t}^{\left(I_{K}\right)}} \\
& \quad=e^{-\left(\rho_{i} b_{i}+\rho_{I_{K}}\left(1-b_{i}\right)\right) u} E_{t}\left[\left(\left(\frac{D_{t+u}}{D_{t}}\right)^{-\gamma_{I_{K}}} \frac{Z_{I_{K} t+u}}{Z_{I_{K}}}\right)^{1-b_{i}}\left(\frac{Z_{i t+u}}{Z_{i t}}\right)^{b_{i}}\right]
\end{aligned}
$$

is independent of $t$.

By Lemma 4.1, we have

$$
M_{t} c_{i t} \sim M_{t}^{\left(I_{K}\right)} c_{i t}^{\left(I_{K}\right)}
$$

and a direct application of (44) and the same argument as in the proof of Proposition 7.2 implies that

$$
E_{t}\left[M_{t+u} c_{i t+u}\right] \sim E_{t}\left[M_{t+u}^{\left(I_{K}\right)} c_{i t+u}^{\left(I_{K}\right)}\right]
$$


By the same argument as in the proof of (28) (but based on the bounds of 44), we get that

$$
\lim _{t \rightarrow \infty} \frac{E_{t}\left[\omega_{j t+u} D_{t+u}^{-1} M_{t+u} c_{i t+u}\right]}{E_{t}\left[M_{t+u} c_{i t+u}\right]} \rightarrow 0
$$

and in complete analogy with the proof of (28), we obtain

$$
E_{t}\left[\theta_{t+u} M_{t+u} c_{i t+u}\right] \sim \theta^{\left(I_{K}\right)} E_{t}\left[M_{t+u} c_{i t+u}\right] .
$$

and from there

$$
\lim _{t \rightarrow \infty} \frac{E_{t}\left[\theta_{t+u} M_{t+u} c_{i t+u}\right]}{M_{t} c_{i t}}=\theta^{\left(I_{K}\right)} \frac{E_{t}\left[M_{t+u}^{\left(I_{K}\right)} c_{i t+u}^{\left(I_{K}\right)}\right]}{M_{t}^{\left(I_{K}\right)} c_{i t}^{\left(I_{K}\right)}}
$$

and

$$
\lim _{t \rightarrow \infty} \frac{E_{t}\left[M_{t+u} c_{i t+u}\right]}{M_{t} c_{i t}}=\frac{E_{t}\left[M_{t+u}^{\left(I_{K}\right)} c_{i t+u}^{\left(I_{K}\right)}\right]}{M_{t}^{\left(I_{K}\right)} c_{i t}^{\left(I_{K}\right)}} .
$$

Now, let us prove that

$$
\lim _{t \rightarrow \infty} \int_{0}^{\infty} \frac{E_{t}\left[\theta_{t+u} M_{t+u} c_{i t+u}\right]}{M_{t} c_{i t}} d u=\theta^{\left(I_{K}\right)} \int_{0}^{\infty} \frac{E_{t}\left[M_{t+u} c_{i t+u}^{\left(I_{K}\right)}\right]}{M_{t} c_{i t}^{\left(I_{K}\right)}} d u
$$

and

$$
\lim _{t \rightarrow \infty} \frac{W_{i t}}{c_{i t}}=\lim _{t \rightarrow \infty} \int_{0}^{\infty} \frac{E_{t}\left[M_{t+u} c_{i t+u}\right]}{M_{t} c_{i t}} d u=\int_{0}^{\infty} \frac{E_{t}\left[M_{t+u} c_{i t+u}^{\left(I_{K}\right)}\right]}{M_{t} c_{i t}^{\left(I_{K}\right)}} d u
$$

By the Lebesgue dominated convergence theorem, it suffices to show that there exists a integrable function $g(u)$ such that

$$
\frac{E_{t}\left[M_{t+u} c_{i t+u}\right]}{M_{t} c_{i t}}=E_{t}\left[\left(\frac{M_{t+u}}{M_{t}}\right)^{1-b_{i}}\left(e^{-\rho u} \frac{Z_{i t+u}}{Z_{i t}}\right)^{b_{i}}\right] \leq g(u) .
$$

By (30) and using the fact that for $\alpha>0$, there exists a constant $K>0$ such that

$$
\left(\sum_{i} x_{i}\right)^{\alpha} \leq K \sum_{i} x_{i}^{\alpha},
$$


we have

$$
\begin{aligned}
& \left(\frac{M_{\tau}}{M_{t}}\right)^{1-b_{i}}\left(e^{-\rho_{i}(\tau-t)} \frac{Z_{i \tau}}{Z_{i t}}\right)^{b_{i}} \\
& \quad \leq K \sum_{j} e^{-\rho_{j}(\tau-t)\left(1-b_{i}\right)}\left(\frac{Z_{j \tau}}{Z_{j t}}\left(\frac{D_{\tau}}{D_{t}}\right)^{-\gamma_{j}}\right)^{1-b_{i}}\left(e^{-\rho_{i}(\tau-t)} \frac{Z_{i \tau}}{Z_{i t}}\right)^{b_{i}} .
\end{aligned}
$$

Now, using the Young inequality

$$
x^{1-b_{i}} y^{b_{i}} \leq\left(1-b_{i}\right) x+b_{i} y
$$

we get that

$$
\begin{gathered}
e^{-\rho_{j}(\tau-t)\left(1-b_{i}\right)}\left(\frac{Z_{j \tau}}{Z_{j t}}\left(\frac{D_{\tau}}{D_{t}}\right)^{-\gamma_{j}}\right)^{1-b_{i}}\left(e^{-\rho_{i}(\tau-t)} \frac{Z_{i \tau}}{Z_{i t}}\right)^{b_{i}} \\
=\left(e^{-\rho_{j}(\tau-t)}\left(\frac{Z_{j \tau}}{Z_{j t}}\left(\frac{D_{\tau}}{D_{t}}\right)^{1-\gamma_{j}}\right)^{1-b_{i}}\left(e^{-\rho_{i}(\tau-t)} \frac{Z_{i \tau}}{Z_{i t}}\left(\frac{D_{\tau}}{D_{t}}\right)^{1-\gamma_{i}}\right)\right)^{b_{i}} \\
\leq K\left(e^{-\rho_{i}(\tau-t)} \frac{Z_{i \tau}}{Z_{i t}}\left(\frac{D_{\tau}}{D_{t}}\right)^{1-\gamma_{i}}+e^{-\rho_{j}(\tau-t)} \frac{Z_{j \tau}}{Z_{j t}}\left(\frac{D_{\tau}}{D_{t}}\right)^{1-\gamma_{j}}\right)
\end{gathered}
$$

and hence

$$
\frac{E_{t}\left[M_{t+u} c_{i t+u}\right]}{M_{t} c_{i t}} \leq K \sum_{j} e^{-u S_{i}^{-1}}
$$

which is integrable by the assumption that $S_{i}>0$. As a direct consequence, we have

$$
\lim _{t \rightarrow \infty} \pi_{i t}=\frac{\delta_{i}+\theta^{\left(I_{K}\right)}}{\sigma \gamma_{i}}
$$

3. We define

$$
f\left(t, W_{t}\right)=E_{t}\left[\int_{t}^{\infty} e^{-\rho_{i} b_{i}(\tau-t)} \frac{Z_{i \tau}^{b_{i}} M_{\tau}^{1-b_{i}}}{Z_{i t}^{b_{i}} M_{t}^{1-b_{i}}}\right] .
$$

Then, the wealth $w_{i t}$ of agent $i$ satisfies

$$
w_{i t}=Z_{i t}^{b_{i}} M_{t}^{-b_{i}} e^{-\rho_{t}} f\left(t, W_{t}\right) .
$$


An applucation of Ito's formula implies that

$$
\pi_{i t} \sigma_{t}=\delta_{i} b_{i}+\theta_{t} b_{i}+\frac{\left(d / d W_{t}\right) f\left(t, W_{t}\right)}{f\left(t, W_{t}\right)}
$$

and hence

$$
\pi_{i t}^{\text {hedging }}=\sigma_{t}^{-1} \frac{\left(d / d W_{t}\right) f\left(t, W_{t}\right)}{f\left(t, W_{t}\right)} .
$$

To determine the sign of the hedging portfolio, we need to check whether $f$ is monotone increasing.

We have

$$
\begin{aligned}
& E_{t}\left[\int_{t}^{\infty} e^{-\rho_{i} b_{i}(\tau-t)} \frac{Z_{i \tau}^{b_{i}} M_{\tau}^{1-b_{i}}}{Z_{i t}^{b_{i}} M_{t}^{1-b_{i}}}\right] \\
= & E_{t}\left[e^{-\rho_{i} b_{i}(\tau-t)-0.5 b_{i} \delta_{i}^{2}(\tau-t)+\delta_{i} b_{i}\left(W_{\tau}-W_{t}\right)-\left(1-b_{i}\right) \int_{t}^{\tau}\left(r_{s}+0.5 \theta_{s}^{2}\right) d s+\int_{t}^{\tau}\left(\delta_{i} b_{i}+\left(b_{i}-1\right) \theta_{s}\right) d W_{s}}\right] \\
& =E_{t}^{P_{i}}\left[\int_{t}^{\infty} e^{-\int_{t}^{\tau} \zeta_{i s} d s} d \tau\right]
\end{aligned}
$$

where $P_{i}$ is a new measure with density process

$$
\frac{d P_{i}}{d P}=e^{-\int_{0}^{t}\left(\delta_{i} b_{i}+\left(b_{i}-1\right) \theta_{s}\right)^{2} d s+\int_{t}^{\tau}\left(\delta_{i} b_{i}+\left(b_{i}-1\right) \theta_{s}\right) d W_{s}}
$$

and

$$
\begin{aligned}
& \zeta_{i t}=\rho_{i} b_{i}+0.5 b_{i} \delta_{i}^{2}+\left(1-b_{i}\right) r_{t}+0.5\left(1-b_{i}\right) \theta_{t}^{2}-0.5\left(\delta_{i} b_{i}+\left(b_{i}-1\right) \theta_{t}\right)^{2} \\
& =\rho_{i} b_{i}+0.5 b_{i}\left(1-b_{i}\right) \delta_{i}^{2}+\left(1-b_{i}\right) r_{t}+0.5\left(1-b_{i}\right) b_{i} \theta_{t}^{2}+\delta_{i} b_{i}\left(1-b_{i}\right) \theta_{t}
\end{aligned}
$$

Thus, it all reduces to checking monotonicity of

$$
\zeta_{i}\left(W_{t}\right)=r_{t}+\frac{1}{2} b_{i} \theta_{t}^{2}+\delta_{i} b_{i} \theta_{t}
$$

Suppose that risk aversion is homogeneous. Then, the derivative of $\zeta_{i}$ is given by

$$
\operatorname{Cov}^{\omega_{i}}\left(b \sigma \delta_{i}+\frac{1}{2} b(1-b) \delta_{i}^{2}+b \rho_{i}, \delta_{i}\right)-2 b^{2}\left(\theta_{t}+\delta_{i}\right) \operatorname{Var}^{\omega_{i}}\left(\delta_{i}\right)
$$

Thus, we get the result (using the fact that $\theta_{t} \in\left[\min _{j} \theta_{j}, \max _{j} \theta_{j}\right]$ ). 


\section{References}

[1] Berrada, T., 2008, Bounded Rationality and Asset Pricing with Intermediate Consumption, Review of Finance, forthcoming.

[2] Berrada, T., Hugonnier, J. and M. Rindisbacher, 2007, Heterogenous Preferences and Equilibrium Trading Volume, Journal of Financial Economics, 83, 719-750.

[3] Bhamra, H. S. and R. Uppal (2009a): "The Effect of Introducing a NonRedundant Deerivative on the Volatility of Stock-Market Returns When Agents Differ in Risk Aversion", forthcoming in Review of Financial Studies.

[4] Bhamra, H. S. and R. Uppal (2009b): "Asset Prices with Heterogeneity in Preferences and Beliefs", working paper.

[5] Blume, L., and D. Easley, 2006, If you are so smart, why aren't you rich. Belief selection in complete and incomplete markets, Econometrica, 74(4), 929-966.

[6] Brunnermeier, M. and S. Nagel, 2008, Do wealth fluctuations generate time varying risk aversion ? Micro Evidence from Individuals' Asset Allocation, American Economic Review 98, 3, 713-736.

[7] Campbell, J.Y., and J.H. Cochrane, 1999, By force of Habit: A Consumption Based Explanation of Aggregate Stock Market Behavior, Journal of Political Economy 107, 2, 205-251.

[8] Cvitanić, J. and S. Malamud, 2009a, Relative Extinction of Heterogeneous Agents.

[9] Cvitanić, J. and S. Malamud, 2009b, Risk Aversion and Equilibrium Optimal Portfolios in Large Markets.

[10] Cvitanić, J. and S. Malamud, 2009c, Equilibrium Driven by Discounted Dividend Volatility. Working Paper.

[11] Detemple J., and S. Murthy, 1997, Equilibrium Asset Prices and NoArbitrage with Portfolio Constraints, Review of Financial Studies, 10, 1133-1174. 
[12] Duffie, D. and C.-F. Huang, 1985, Implementing Arrow-Debreu Equilibria by Continuous Trading of Few Long-Lived Securities, Econometrica, 53, $1337-1356$

[13] Dumas, B., 1989, Two-person dynamic equilibrium in the capital market, Review of Financial Studies, 2, 157-188.

[14] Dumas, Bernard, Alexander Kurshev and Raman Uppal (2008): "Equilibrium Portfolio Strategies in the Presence of Sentiment Risk and Excess Volatility," forthcoming in Journal of Finance.

[15] Jouini, E. , Marin, J.-M. and C. Napp, 2008, Discounting and Divergence of Opinion, to appear Journal of Economic Theory.

[16] Jouini, E. and C. Napp, 2007, Consensus consumer and intertemporal asset pricing under heterogeneous beliefs, Review of Economic Studies, 74, 1149-1174.

[17] Jouini, E. and C. Napp, 2009, Unbiased Disagreement and the Efficient Market Hypothesis, Working Paper.

[18] Kogan, L., Ross, S., Wang, J., and M. Westerfield, 2006, The Price Impact and Survival of Irrational Traders, Journal of Finance, 61, 195229 .

[19] Kogan, L., Ross, S., Wang, J., and M. Westerfield, 2008, Market Selection, Working Paper.

[20] Mele, A., 2007, Asymmetric Stock Market Volatility and the Cyclical Behavior of Expected Returns, Journal of Financial Economics, 86, 446478 .

[21] Modigliani, F. and R. Sutch, 1966, Innovations in Interest Rate Policy, American Economic Review, 56, 178-197.

[22] Riedel, F., 2001, Existence of Arrow-Radner Equilibrium with Endogenously Complete Markets with Incomplete Information, Journal of Economic Theory, 97, 109-122.

[23] Wang, J., 1996, The Term Structure of Interest Rates In A Pure Exchange Economy With Heterogeneous Investors, Journal of Financial Economics 41, 75-110. 
[24] Yan, H., 2008, Natural Selection in Financial Markets: Does It Work?, Management Science, 54, 1935-1950. 


\section{swiss : finance: institute}

c/o University of Geneva

40 bd du Pont d'Arve

1211 Geneva 4

Switzerland

$\mathrm{T}+41223798471$

F +41 223798277

RPS@sfi.ch

www.SwissFinanceInstitute.ch 